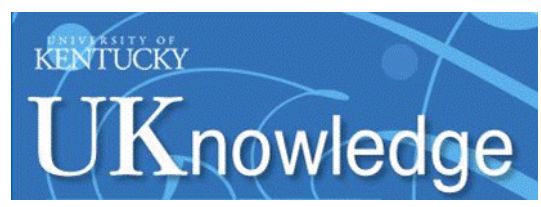

University of Kentucky

UKnowledge

4-2-2018

\title{
SDSS-IV MaNGA: Star Formation Cessation in Low-Redshift Galaxies. I. Dependence on Stellar Mass and Structural Properties
}

\author{
Enci Wang \\ University of Science and Technology of China, China \\ Cheng Li \\ Tsinghua University, China \\ Ting Xiao \\ Shanghai Astronomical Observatory, China \\ Lin Lin \\ Shanghai Astronomical Observatory, China \\ Matthew Bershady \\ University of Wisconsin - Madison
}

See next page for additional authors

Follow this and additional works at: https://uknowledge.uky.edu/physastron_facpub

Part of the Astrophysics and Astronomy Commons

Right click to open a feedback form in a new tab to let us know how this document benefits you.

\section{Repository Citation}

Wang, Enci; Li, Cheng; Xiao, Ting; Lin, Lin; Bershady, Matthew; Law, David R.; Merrifield, Michael; Sanchez, Sebastian F.; Riffel, Rogemar A.; Riffel, Rogerio; and Yan, Renbin, "SDSS-IV MaNGA: Star Formation Cessation in Low-Redshift Galaxies. I. Dependence on Stellar Mass and Structural Properties" (2018). Physics and Astronomy Faculty Publications. 587.

https://uknowledge.uky.edu/physastron_facpub/587

This Article is brought to you for free and open access by the Physics and Astronomy at UKnowledge. It has been accepted for inclusion in Physics and Astronomy Faculty Publications by an authorized administrator of UKnowledge. For more information, please contact UKnowledge@lsv.uky.edu. 
SDSS-IV MaNGA: Star Formation Cessation in Low-Redshift Galaxies. I. Dependence on Stellar Mass and Structural Properties

\author{
Digital Object Identifier (DOI) \\ https://doi.org/10.3847/1538-4357/aab263 \\ Notes/Citation Information \\ Published in The Astrophysical Journal, v. 856, no. 2, 137, p. 1-22. \\ (C) 2018. The American Astronomical Society. All rights reserved.
}

The copyright holder has granted the permission for posting the article here.

Authors

Enci Wang, Cheng Li, Ting Xiao, Lin Lin, Matthew Bershady, David R. Law, Michael Merrifield, Sebastian F. Sanchez, Rogemar A. Riffel, Rogerio Riffel, and Renbin Yan 


\title{
SDSS-IV MaNGA: Star Formation Cessation in Low-redshift Galaxies. I. Dependence on Stellar Mass and Structural Properties
}

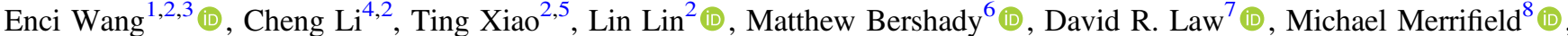 \\ Sebastian F. Sanchez ${ }^{9}$ (1), Rogemar A. Riffel ${ }^{10,11}$, Rogerio Riffel ${ }^{11,12}$, and Renbin Yan ${ }^{13}$ (1) \\ ${ }^{1}$ CAS Key Laboratory for Research in Galaxies and Cosmology, Department of Astronomy, University of Science and Technology of China, \\ Hefei 230026, People's Republic of China; ecwang16@ustc.edu.cn \\ ${ }^{2}$ Shanghai Astronomical Observatory, Nandan Road 80, Shanghai 200030, People's Republic of China \\ ${ }^{3}$ School of Astronomy and Space Science, University of Science and Technology of China, Hefei 230026, People's Republic of China \\ ${ }^{4}$ Tsinghua Center for Astrophysics and Physics Department, Tsinghua University, Beijing 100084, People's Republic of China; cli2015@tsinghua.edu.cn \\ 5 Department of Physics, Zhejiang University, Hangzhou 310027, People's Republic of China \\ ${ }^{6}$ Department of Astronomy, University of Wisconsin-Madison, 475 N. Charter Street, Madison, WI 53706, USA \\ ${ }_{8}^{7}$ Space Telescope Science Institute, 3700 San Martin Drive, Baltimore, MD 21218, USA \\ ${ }^{8}$ School of Physics \& Astronomy, University of Nottingham, Nottingham NG7 2RD, UK \\ ${ }^{9}$ Instituto de Astronomía, Universidad Nacional Autónoma de México, A. P. 70-264, C.P. 04510, México, D.F., Mexico \\ ${ }^{10}$ Departamento de Física, Centro de Ciências Naturais e Exatas, Universidade Federal de Santa Maria, 97105-900, Santa Maria, RS, Brazil \\ ${ }^{11}$ Laboratório Interinstitucional de e-Astronomia-LIneA, Rua Gal. José Cristino 77, Rio de Janeiro, RJ-20921-400, Brazil \\ ${ }^{12}$ Departamento de Astronomia, Instituto de Física, Universidade Federal do Rio Grande do Sul, CP 15051, 91501-970, Porto Alegre, RS, Brazil \\ ${ }^{13}$ Department of Physics and Astronomy, University of Kentucky, 505 Rose Street, Lexington, KY 40506, USA \\ Received 2017 October 20; revised 2018 January 19; accepted 2018 February 24; published 2018 April 2
}

\begin{abstract}
We investigate radial gradients in the recent star formation history (SFH) of 1917 galaxies with $0.01<z<0.14$ from the Mapping Nearby Galaxies at Apache Point Observatory project. For each galaxy, we obtain twodimensional maps and radial profiles for three spectroscopically measured parameters that are sensitive to the recent $\mathrm{SFH}: \mathrm{D}_{n}(4000)$ (the $4000 \AA$ break), $\mathrm{EW}\left(\mathrm{H} \delta_{A}\right)$, and $\mathrm{EW}(\mathrm{H} \alpha)$ (the equivalent width of the $\mathrm{H} \delta$ absorption and the $\mathrm{H} \alpha$ emission line). The majority of the spaxels are consistent with models of a continuously declining star formation rate, indicating that starbursts occur rarely in local galaxies with regular morphologies. We classify the galaxies into three classes: fully star-forming (SF), partly quenched (PQ), and totally quenched (TQ). The galaxies that are less massive than $10^{10} M_{\odot}$ present at most weak radial gradients in the diagnostic parameters. In contrast, massive galaxies with a stellar mass above $10^{10} M_{\odot}$ present significant gradients in the three diagnostic parameters if they are classified as SF or PQ but show weak gradients in $\mathrm{D}_{n}(4000)$ and $\mathrm{EW}\left(\mathrm{H} \delta_{A}\right)$ and no gradients in $\mathrm{EW}(\mathrm{H} \alpha)$ if they are in the TQ class. This implies the existence of a critical stellar mass $\left(\sim 10^{10} M_{\odot}\right)$ above which the star formation in a galaxy is shut down from the inside out. Galaxies tend to evolve synchronously from the inner to the outer regions before their mass reaches the critical value. We have further divided the sample at a fixed mass by both bulge-to-total luminosity ratio and morphological type, finding that our conclusions hold regardless of these factors; it appears that the presence of a central dense object is not a driving parameter but rather a by-product of the star formation cessation process.
\end{abstract}

Key words: galaxies: evolution - galaxies: formation - galaxies: general - galaxies: stellar content - methods: observational - surveys

\section{Introduction}

The fraction of red quiescent galaxies in the universe has gradually but significantly increased since $z \sim 1$ (e.g., Bell et al. 2004; Bundy et al. 2006; Faber et al. 2007), implying that the population of blue star-forming (SF) galaxies at high redshift has largely evolved to the present-day red population. The cessation of star formation is thus a driving process for galaxy evolution over the past $\sim 8$ Gyr. A complete picture of the way in which star formation is shut down remains elusive, however, although extensive studies, both observational and theoretical, have been carried out in past decades. These studies have proposed and examined a variety of physical processes, finding that both the external environment and internal structural properties play important roles in star formation cessation in galaxies.

For central galaxies located at the center of massive dark halos with a halo mass exceeding a critical mass of $\sim 10^{12} M_{\odot}$, shock heating may effectively suppress the gas cooling efficiency, thus preventing further star formation (e.g., Rees
\& Ostriker 1977; Silk 1977; Blumenthal et al. 1984; Birnboim \& Dekel 2003; Kereš et al. 2005; Cattaneo et al. 2006; Dekel \& Birnboim 2006). In this case, accretion of the hot halo gas onto the central supermassive black hole in central galaxies is expected to trigger "radio-mode" active galactic nucleus (AGN) feedback, commonly adopted as one of the quenching ${ }^{14}$ processes in semi-analytic models (e.g., Croton et al. 2006). For central galaxies hosted by less massive halos, processes internal to the systems are more important. Observational studies have clearly established that a massive dense structure at the galactic center (such as a prominent bulge) is present in the majority of quenched central galaxies at both low and high redshifts (Bell 2008; Bundy et al. 2010; Masters et al. 2010; Bell et al. 2012; Cheung et al. 2012; Fang et al. 2013; Bluck et al. 2014; Barro et al. 2017). This finding was initially

\footnotetext{
14 We note that in this paper, "quenching" does not specifically mean a rapid shutdown of star formation. In most cases, we use "cessation" instead of "quenching," thus including both rapid shutdown and slow diminution over long timescales.
} 
interpreted as observational evidence for AGN feedback quenching star formation in central galaxies (e.g., Bell 2008) and was later also thought of as a natural consequence of the "morphology quenching" picture, in which a massive central object is able to stabilize the rotating disk against star formation (e.g., Martig et al. 2009).

In contrast, star formation cessation in satellite galaxies appears to be primarily driven by external processes occurring within or around the host groups. These processes include gas stripping by tidal interactions (Toomre \& Toomre 1972; Moore et al. 1996) and ram pressure (Gunn \& Gott 1972; Abadi et al. 1999; Vogt et al. 2004; Chung et al. 2009; Merluzzi et al. 2013), galaxy mergers (e.g., Lin et al. 2014), and more gentle processes such as "starvation" or "strangulation" (e.g., Balogh et al. 2000; Weinmann et al. 2009; Peng et al. 2015). The importance and strength of these environmental effects depend on a variety of factors: the dark matter mass of the host halos, the clustercentric radius of galaxy locations, galaxy stellar mass, and, particularly, the structural parameters of galaxies (e.g., surface stellar mass density; Li et al. 2012; Zhang et al. 2013).

Therefore, in order to have a full understanding of star formation cessation in galaxies, one would need spatially resolved spectroscopy to measure the stellar and gaseous components for a large sample of galaxies covering a wide range of global properties (e.g., stellar mass, color, star formation rate [SFR], morphology, nuclear activity, etc.) and probing environmental conditions over a wide range of spatial scales (tidal interactions/mergers, central/satellite classification, local density, dark halo mass, halo assembly history, large-scale structure configuration, etc.). Resolved spectroscopy has become available only recently thanks to the integral field unit (IFU) surveys accomplished in the past decade: SAURON (Bacon et al. 2001; de Zeeuw et al. 2002), DiskMass (Bershady et al. 2010), ATLAS $^{3 \mathrm{D}}$ (Cappellari et al. 2011), CALIFA (Sánchez et al. 2012), VENGA (Blanc et al. 2013), SLUGGS (Brodie et al. 2014), and MASSIVE (Ma et al. 2014). These IFU surveys have typically targeted tens or hundreds of local galaxies with different morphological types, enabling detailed studies of the spatially resolved stellar populations and kinematics of galaxies. Meanwhile, the $\mathrm{KMOS}^{3 \mathrm{D}}$ survey is extending the IFU efforts to higher redshifts, observing 600 galaxies at $0.7<z<2.7$ using KMOS at the Very Large Telescope (Wisnioski et al. 2015).

These IFU surveys have enabled two-dimensional maps of star formation and mass assembly histories to be studied with high accuracy for galaxies in the local universe. For instance, the CALIFA survey has spatially resolved light-weighted stellar age, SFR, oxygen abundance, stellar metallicity, and the history of stellar mass assembly for a few hundred galaxies with different morphologies and masses, revealing an inside-out growth picture for massive galaxies and a transition to outside-in growth at $M_{*} \sim 10^{10} M_{\odot}$ (Pérez et al. 2013; González Delgado et al. 2014, 2015, 2016; Sánchez et al. 2014; Sánchez-Blázquez et al. 2014; Catalán-Torrecilla et al. 2017). Specifically, for massive galaxies, the intensity of the SFR is found to decline as one goes from the galactic center outward, suggesting that the cessation of star formation happens from the inside out. This inside-out picture of star formation cessation is broadly consistent with previous studies, which have been based on either multiwavelength broadband photometry (e.g., de Jong 1996; Abraham et al. 1999; Bell \& de Jong 2000; Muñoz-Mateos et al. 2007; Zibetti et al. 2009; Roche et al. 2010; Suh et al. 2010; Tortora et al. 2010, 2011; Gonzalez-Perez et al. 2011;
Szomoru et al. 2012, 2013; Wuyts et al. 2012, 2013; Kauffmann 2015) or long-slit spectroscopy (e.g., Moran et al. 2012; Huang et al. 2013).

More recent IFU surveys have started to construct large statistical samples consisting of more than a thousand galaxies at low $z$. The SAMI survey (Croom et al. 2012) is observing 3400 galaxies using a multiplexed fiber-IFU instrument at the Australian Astronomical Observatory. The Mapping Nearby Galaxies at Apache Point Observatory (MaNGA; Bundy et al. 2015) project is making another big step forward by surveying 10,000 galaxies selected from the Sloan Digital Sky Survey (SDSS) spectroscopic sample. The MaNGA sample spans a wide dynamic range in stellar mass, color, SFR, and environment. More importantly, MaNGA has effective étendue in the near-UV (see Bundy et al. 2015), allowing precise measurements of spectral indices such as the $4000 \AA$ break $\left(\mathrm{D}_{n}(4000)\right)$ and the equivalent width of the $\mathrm{H} \delta$ absorption line $\left(\mathrm{H} \delta_{A}\right)$, indicative of young stellar populations formed in the past 0.1-1 Gyr (Kauffmann et al. 2003). These stellar indices, together with the $\mathrm{H} \alpha$ emission-line equivalent width (EW $(\mathrm{H} \alpha)$ ), provide powerful diagnostics of the recent star formation history (SFH) of a given galactic region. MaNGA thus has unique statistical power in studying star formation cessation in galaxies, and a comprehensive picture of the star formation cessation process may be built up by measuring gradients in these diagnostics for the large sample of galaxies from MaNGA.

Li et al. (2015; hereafter Paper I) presented a demonstration of MaNGA's unique potential in studies of star formation cessation, analyzing the radial profiles of $\mathrm{D}_{n}(4000), \operatorname{EW}\left(\mathrm{H} \delta_{A}\right)$, and $\mathrm{EW}(\mathrm{H} \alpha)$ for 12 galaxies observed in the MaNGA prototype run (P-MaNGA; see Bundy et al. 2015). The radial variation in these diagnostics is found to depend strongly on the star formation status of the galactic center: a centrally SF (CSF) galaxy generally shows no or very weak radial variation across the whole galaxy, while centrally quiescent (CQ) galaxies present significant gradients in all the diagnostics, supporting the "inside-out" picture in which the cessation of star formation propagates from the center of a galaxy outward. In a parallel paper, Wilkinson et al. (2015) derived gradients in stellar age and metallicity by performing full spectral fitting to the P-MaNGA data, finding similar negative gradients in stellar age in CQ galaxies. Using a representative sample of 721 galaxies from the MaNGA internal data release, MaNGA Product Launches 4 (equivalent to the SDSS Data Release 13 [DR13]; SDSS Collaboration et al. 2017), Goddard et al. (2017) extended the P-MaNGA analysis of Wilkinson et al. (2015) and found that the gradients in stellar age tend to be shallow for both early-type and late-type galaxies, with, on average, a positive mass-weighted age gradient $\left(\sim 0.09 \mathrm{dex} / \mathrm{R}_{\mathrm{e}}\right)$ for early-type galaxies and a negative light-weighted age gradient $\left(\sim-0.11 \mathrm{dex} / \mathrm{R}_{\mathrm{e}}\right)$ for late-type galaxies.

This paper is the first of a series of papers in which we will extensively study the star formation cessation process in lowredshift galaxies as observed in MaNGA. In this paper, we extend the analysis of Paper I by using a much larger sample of 1917 galaxies from the MaNGA Product Launches 5 (MPL5, equivalent to the SDSS Data Release 14 [DR14]; Abolfathi et al. 2017). Following Paper I, we use $\mathrm{D}_{n}(4000), \mathrm{EW}\left(\mathrm{H} \delta_{A}\right)$, and $\mathrm{EW}(\mathrm{H} \alpha)$ to trace the SFH over the last 1-2 Gyr for each spaxel in the MaNGA data cubes, thus obtaining both twodimensional maps and one-dimensional radial profiles of the 
recent SFH diagnostics for the galaxies. When compared to those in Paper I, both the sample size and the diagnostic parameter measurements are largely improved, enabling us to measure the gradients in the recent SFH over a wide dynamic range in galaxy mass, color, and structural properties. Based on these measurements, we propose a new quantity, $f_{Q}$, which is the fraction of the quenched area within $1.5 \mathrm{R}_{\mathrm{e}}$, to quantify the global status of the star formation in a galaxy. Accordingly, we classify our galaxies into three subsets at different stages in terms of global star formation: fully SF, partly quenched (PQ), and totally quenched (TQ). We compare this new classifier with both global colors and $\mathrm{D}_{n}(4000)$ at the galactic center measured from SDSS $3^{\prime \prime}$ fiber spectroscopy, which has been widely adopted for the same purpose in previous studies (e.g., Kauffmann et al. 2003, 2007; Cheung et al. 2012; Pan et al. 2014; Guo et al. 2016). We analyze the gradients in the recent SFH for the three types of galaxies, particularly examining the dependence on stellar mass and structural parameters such as the bulge-to-disk ratio. As we will show, these analyses provide interesting implications for the star formation cessation process, which is found to depend strongly on the stellar mass of the galaxy. In following papers of this series, we will further investigate the roles of galactic bars, galaxy-galaxy interactions/mergers, and the large-scale environment in regulating the star formation cessation of galaxies. We will also study the cold gas content of these galaxies by performing follow-up observations of the atomic and molecular gas emission. Throughout this paper, we assume a flat cold dark matter cosmology model with $\Omega_{m}=0.27$, $\Omega_{\Lambda}=0.73$, and $h=0.7$ when computing distance-dependent parameters.

\section{Data}

\subsection{MaNGA Overview}

As one of the three major experiments of the fourth generation of the SDSS (SDSS-IV; Blanton et al. 2017), MaNGA is obtaining integral field spectroscopy for 10,000 nearby galaxies with redshifts in the range $0.01<z<0.15$ (Bundy et al. 2015). The MaNGA instrument utilizes the two dual-channel BOSS spectrographs at the $2.5 \mathrm{~m}$ Sloan Telescope (Gunn et al. 2006; Smee et al. 2013), simultaneously covering wavelengths of over 3600-10300 $\AA$ at $R \sim 2000$ and reaching a target $r$-band $\mathrm{S} / \mathrm{N}=4-8\left(\AA^{-1}\right.$ per $2^{\prime \prime}$ fiber $)$ at $1-2 \mathrm{R}_{\mathrm{e}}$, with a typical integration time of $3 \mathrm{hr}$. The MaNGA IFU includes 29 fiber bundles for the Sloan $3^{\circ}$ diameter field of view, including 12 seven-fiber "mini-bundles" dedicated to flux calibration and 17 science bundles with five different sizes ranging from 19 to 127 fibers, corresponding to on-sky diameters of between $12^{\prime \prime}$ and 32". Details of the MaNGA instrument design, testing, and assembly can be found in Drory et al. (2015).

The MaNGA raw data are reduced with the Data Reduction Pipeline developed by Law et al. $(2015,2016)$. The reduced data for each galaxy are provided in the form of a data cube with a spaxel size of $0 . " 5$, which was chosen as a suitable compromise between the galaxy spatial information and the data volume. The typical effective spatial resolution of the reconstructed data cubes can be described by a Gaussian with a FWHM $\sim 2$ ". 5. Flux calibration mainly corrects for flux loss due to atmospheric absorption and instrument response. With the help of the mini-bundles, the absolute flux calibration is better than $5 \%$ for more than $89 \%$ of MaNGA's wavelength range. The flux calibration, survey strategy, and data-quality tests are described in detail in Yan et al. (2016a, 2016b).

Wake et al. (2017) presented the MaNGA sample design and optimization, which were developed to simultaneously optimize the MaNGA IFU size distribution, the IFU allocation strategy, and the target density. Three samples are defined. The primary and secondary samples are selected to have a flat distribution of the $i$-band absolute magnitude, $M_{i}$, with the assigned IFU bundles reaching 1.5 and 2.5 effective radii, respectively. Supplemental to the primary sample, the colorenhanced sample increases the fraction of rare populations in the color-magnitude diagram, such as high-mass blue galaxies, low-mass red galaxies, and "green-valley" galaxies, by extending the redshift limits in the appropriate color bins.

An overview of the MaNGA instrument, survey design, key science goals, and prototype observations (P-MaNGA) are presented in Bundy et al. (2015). The first MaNGA data release accompanied the SDSS DR13 (SDSS Collaboration et al. 2017), including the reduced MaNGA data cubes for 1390 galaxies observed between 2014 July and 2015 July. The SDSS DR14 (Abolfathi et al. 2017) came out more recently and provides MaNGA data cubes for a total of 2812 galaxies observed by 2016 July. Two value-added catalogs based on MaNGA data were released together with the SDSS DR14; they were contributed by the scientists within the MaNGA team and contain spatially resolved and integrated stellar population properties derived by performing a full spectral fitting of the MaNGA data cubes (Sánchez et al. 2016a; Goddard et al. 2017).

\subsection{SDSS Perspective of the MPL5 Sample}

In this work, we make use of the MPL5 (version-2-0-1 of the MaNGA Product Launches) data, which are equivalent to the MaNGA sample released with SDSS DR14, including a total of 2778 galaxies. We restrict ourselves to galaxies with regular shapes, thus excluding those with irregular/disturbed morphologies (13.2\% of the galaxies are excluded for this reason); these will be studied in a separate paper in this series (T. Xiao et al. 2018 , in preparation). The morphological type of each galaxy is identified by visually examining the $r$-band image from SDSS. We classify the sample galaxies into three broad classes: disklike, spheroid-like, and irregular. We have compared our classifications with those from the Galaxy Zoo project (Willett et al. 2013). Among the irregular/disturbed galaxies in our case, only $13.4 \%$ are classified as having a regular morphology with the voted odd fraction $<0.3$ in Galaxy Zoo, indicating that the two classifications are broadly consistent. We find that almost all the spheroid-like galaxies $(97.8 \%)$ in our classification are smooth-like in Galaxy Zoo. However, our spheroidlike classification is much stricter than the smooth-like classification in Galaxy Zoo, since $38.2 \%$ of the smooth-like galaxies in Galaxy Zoo are classified as disklike according to our classification. We have visually examined the galaxies with discrepant classifications and verified that they do have spirallike features in their image. Furthermore, we exclude 475 galaxies from the color-enhanced sample and keep only those from the primary and secondary samples, considering that the $1 / V_{\max }$ weighting scheme is applicable only to these samples (see Wake et al. 2017). Finally, we exclude 19 galaxies with apparent problems in data reduction or spectral fitting. These restrictions yield a sample of 1917 galaxies in total. We take 

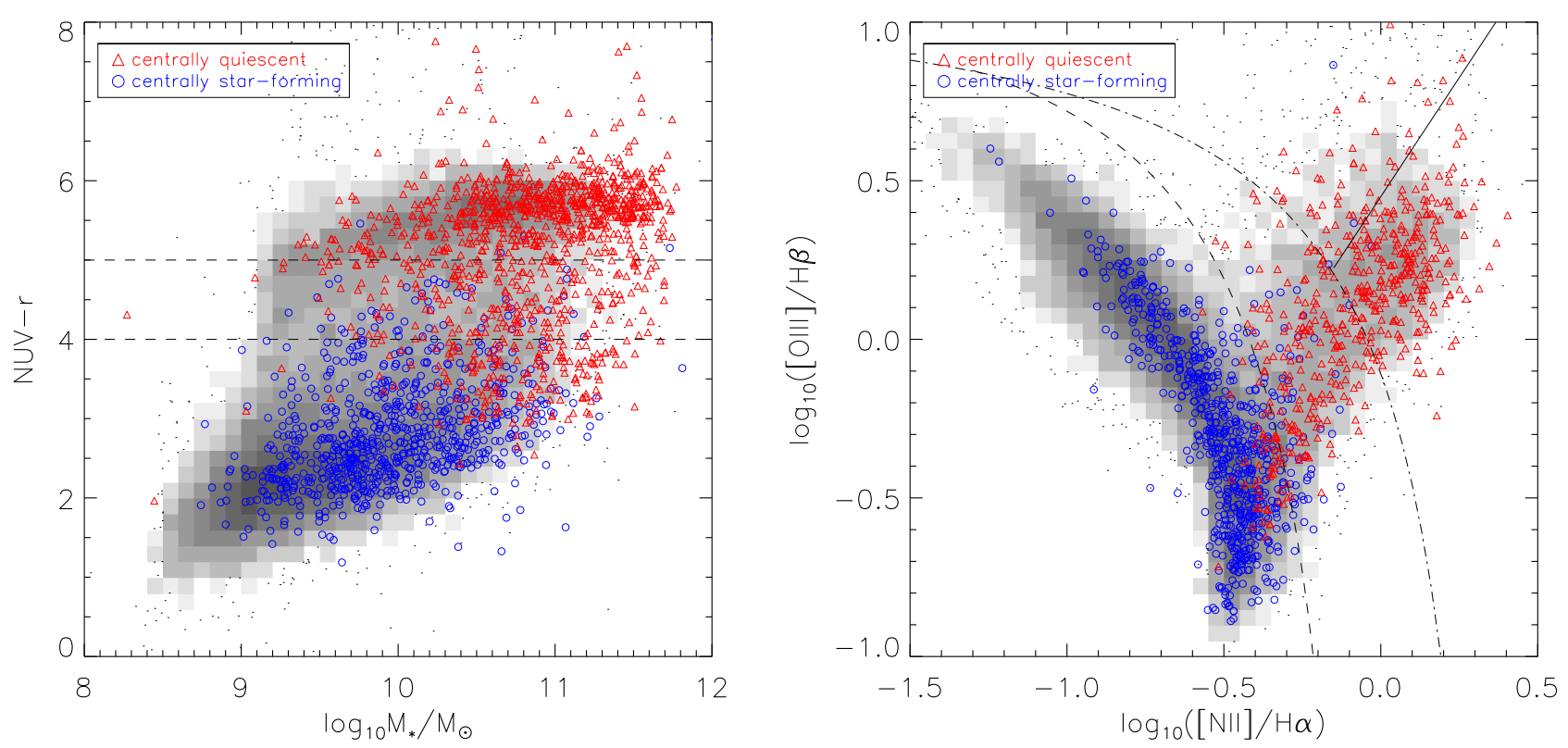

Figure 1. CQ (red triangles) and CSF (blue circles) galaxies plotted on the stellar mass vs. near-UV $-r$ plane (left panel) and the diagram of Baldwin et al. (1981; right panel). For comparison, a volume-limited sample selected from SDSS is shown in the background in both panels. The two horizontal lines in the left panel indicate near-UV $-r=4$ and 5. In the right panel, the dashed and dash-dotted curves are adopted from Kauffmann et al. (2003) and Kewley et al. (2006) to separate the AGN from the SF galaxies.

this as our full sample, and all of the analyses below are based on this sample.

In what follows, we correct the sample selection effects with the $1 / V_{\max }$ weighting method following the prescription in Wake et al. (2017). For a given galaxy in the primary or secondary sample with an SDSS $i$-band magnitude of $M_{i}$, Wake et al. (2017) calculated the minimum redshift $Z_{\min }$ and the maximum redshift $Z_{\max }$ over which the galaxy can be included in the MaNGA target sample. This corresponds to a maximum volume, $V_{\max }$. The MaNGA sample selection effects are then corrected by weighting each galaxy by $1 / V_{\max }$ when performing statistical analyses. The weights are multiplied by a factor of $1 / 0.769$ for galaxies from the secondary sample, which is randomly selected from the target sample at a rate of $76.9 \%$ for real observations. We note that we have ignored a slight selection bias in angular size caused by the fact that, for multiple reasons, the MaNGA IFU size distribution does not exactly match the targeted distribution (see Section 6.2 in Wake et al. 2017 for details), which is expected to have no significant effect on our analyses. Our sample covers a redshift range of $0.01<z<0.14$, corresponding to a cosmic time interval of $1.6 \mathrm{Gyr}$. We assume that the cosmic evolution of our measurements is negligible. In fact, we have repeated the main analyses to be presented in the rest of the paper while limiting the sample to a narrower redshift range of $z<0.06$ and found the results remain essentially unchanged.

Before analyzing the MaNGA data cubes, we examine the global properties of our sample using SDSS single-fiber spectroscopy and broadband photometry. Figure 1 displays the galaxies on the plane of stellar mass versus near-UV $-r$ color, as well as the BPT diagram (Baldwin et al. 1981). The stellar mass and near-UV $-r$ color are taken from the NASA Sloan Atlas (NSA), ${ }^{15}$ which is a catalog constructed by Blanton et al. (2011) listing the physical parameters for more than 640,000 local

\footnotetext{
15 http://www.nsatlas.org
}

galaxies based on data from GALEX, SDSS, and the Two-Micron All Sky Survey. The relevant emission-line ratios used for the BPT diagram are taken from the MPA/JHU SDSS database ${ }^{16}$ (Brinchmann et al. 2004). In the BPT diagram, the dashed and dash-dotted curves are adopted from Kauffmann et al. (2003) and Kewley et al. (2006) to separate the AGN from the SF galaxies. For comparison, we have selected a volume-limited sample from the NSA consisting of 35,070 galaxies with an $r$ band of an absolute magnitude brighter than -17.2 and redshift between 0.01 and 0.03 . Distributions of this sample are shown as a gray-scale background in both panels.

Figure 2 shows the relations between the three $\mathrm{SFH}$ diagnostics: $\mathrm{D}_{n}(4000), \mathrm{EW}\left(\mathrm{H} \delta_{A}\right)$, and $\mathrm{EW}(\mathrm{H} \alpha)$. These parameters are obtained from the SDSS 3 " 0 fiber spectra, so they combine to probe the recent SFH of the central $1-2 \mathrm{kpc}$ of the MaNGA galaxies. We take the measurements of these parameters from the MPA/JHU database. As before, distributions of the volume-limited NSA sample are plotted as a grayscale background. For comparison, we also show the predicted relations of the same parameters by the Bruzual \& Charlot (2003) models of solar metallicity that follow exponentially declining SFHs (SFR $\propto \exp (-t / \tau)$ ). The solid lines are for continuous star formation decline with $\tau>5 \times 10^{8} \mathrm{yr}$, and the dashed lines are for star formation bursts with $\tau<5 \times 10^{8} \mathrm{yr}$. Different lines represent the different characteristic timescales $\tau$. The $\mathrm{H} \alpha$ luminosity is computed by converting Lyman continuum photons to $\mathrm{H} \alpha$ following Hunter \& Elmegreen (2004; see Equations B2-B4 in their Appendix), adopting the recombination coefficients and $\mathrm{H} \alpha / \mathrm{H} \beta$ ratios from Hummer \& Storey (1987).

Figure 1 shows that our sample well represents the general population of galaxies at a stellar mass above $\sim 10^{9} M_{\odot}$, covering the full parameter space in both the color-mass diagram and the BPT diagram. Our sample lacks galaxies

\footnotetext{
${ }^{16}$ http://www.mpa-garching.mpg.de/SDSS/DR7/
} 

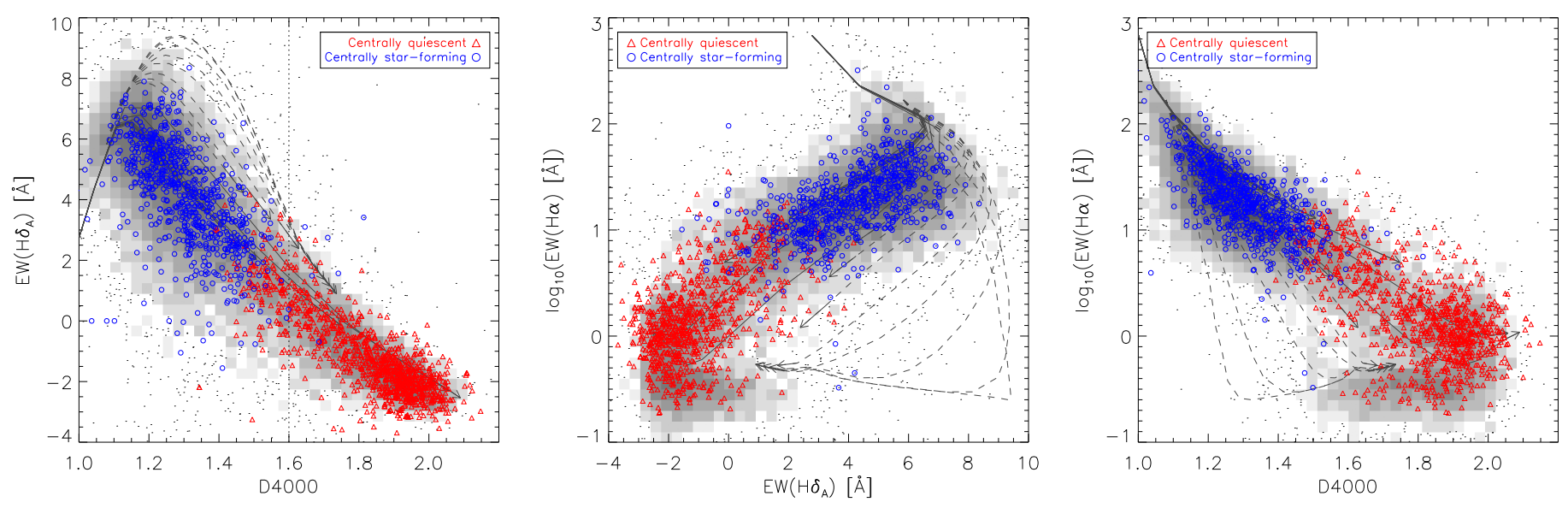

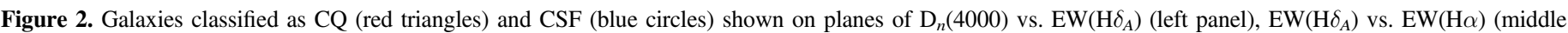

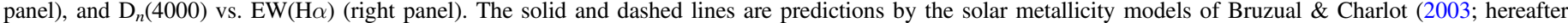

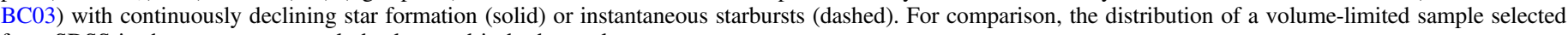
from SDSS is shown as a gray-scale background in both panels.

below $\sim 10^{9} M_{\odot}$ and galaxies with the lowest [N II] $/ \mathrm{H} \alpha$, which can both be attributed to the faint limit of $M_{i}$ adopted for the MaNGA target sample (see Wake et al. 2017). In addition, our sample appears to have a surfeit of the most massive galaxies as compared to the volume-limited NSA sample, which is a consequence of the MaNGA sample design that attempts to make the sampling uniform in mass (Wake et al. 2017). From Figure 2, we see that the central regions of the galaxies form relatively tight relations involving all three diagnostics, which closely follow the regions of the continuous star formation models.

Following Paper I, we divide the galaxies into two subsamples according to the central $\mathrm{D}_{n}(4000)$ measured from the central spaxel in the MaNGA data cube ${ }^{17}$ : CQ (red triangles) galaxies with $\mathrm{D}_{n}(4000)_{\text {cen }}>1.6$ and CSF (blue circles) galaxies with $\mathrm{D}_{n}(4000)_{\text {cen }} \leqslant 1.6$ (Li et al. 2015). As can be seen from Figure 1, almost all CSF galaxies are located in the blue cloud with near$\mathrm{UV}-r<4$, and the majority of CQ galaxies are located in the red sequence with near-UV $-r>5$. However, a large fraction of CQ galaxies fall in the green valley, and some of the high-mass galaxies $\left(\log _{10}\left(M_{*} / M_{\odot}\right)>10.0\right)$ are found even in the blue cloud. This indicates that high-mass galaxies may be still forming stars if seen as a whole, even though the galactic center is already quenched.

\subsection{Full Spectral Fitting}

We perform a full spectral fitting for each spaxel in the MaNGA data cubes following the same method as described in Paper I and obtain two-dimensional maps of $\mathrm{D}_{n}(4000)$, $\mathrm{EW}\left(\mathrm{H} \delta_{A}\right)$, and $\mathrm{EW}(\mathrm{H} \alpha)$ for each galaxy. We use the empirical spectral fitting code developed by $\mathrm{Li}$ et al. (2005), which utilizes a set of nine template spectra constructed from observed stellar and galactic spectra using the technique of principal component analysis. The median $\chi^{2}$ of the spectral fitting is 1.12 for the whole sample, with only $3.7 \%$ of the spaxels fitted with $\chi^{2}>3$. We have visually examined these spectra and find that the large $\chi^{2}$ has multiple causes, but the regions of the $\mathrm{D}_{n}(4000)$ and $\mathrm{EW}\left(\mathrm{H} \delta_{A}\right)$ are indeed well fitted in

\footnotetext{
${ }^{17}$ Note that the CFS and CQ classifications are based on the MaNGA central spaxel, while the parameters used for making Figure 2 come from SDSS singlefiber spectroscopy. This is why there is not a sharp break between the red and blue symbols in the figure.
}

most cases. Refer to Li et al. (2005) for a detailed description of the construction of the templates and tests of the spectral fitting results. We then measure $\mathrm{D}_{n}(4000)$ and $\mathrm{EW}\left(\mathrm{H} \delta_{A}\right)$ based on the best-fitting stellar spectra without correcting the intrinsic dust attenuation, as well as the $\operatorname{EW}(\mathrm{H} \alpha)$ from the pure emission-line component, obtained by subtracting the best-fit stellar component from the observed spectrum. We fit the $\mathrm{H} \alpha$ emission line with a single Gaussian profile when measuring $\mathrm{EW}(\mathrm{H} \alpha)$. Examples of spectral fits and maps of the three diagnostics, as well as tests on the methodology, can be found in Paper I. We also use the public spectral fitting code STARLIGHT (Cid Fernandes et al. 2005) to derive a stellar mass for each spaxel, which will be used to obtain massweighted parameters.

\section{Results}

\subsection{A Single Classifier of Star Formation Status}

Previous studies usually used broadband colors (e.g., NUV $-r$ ) or spectroscopic diagnostics measured at galactic centers (SDSS-based SFR and $\mathrm{D}_{n}(4000)$ ) to divide galaxies into SF and quiescent subsamples. As shown above, however, the central $\mathrm{D}_{n}(4000)$ and global NUV $-r$ color provide inconsistent classifications in many cases. Here we introduce a new parameter to characterize the overall status of star formation in a galaxy based on two-dimensional maps of $\mathrm{D}_{n}(4000)$ and $\mathrm{EW}(\mathrm{H} \alpha)$. The parameter, $f_{Q}$, is defined as the mass-weighted fraction of spaxels that are quenched within a given galactic radius $R$,

$$
f_{Q}(R)=\frac{\sum_{r<R} m_{*, i} \times f_{i}\left(\mathrm{D}_{n}(4000), \mathrm{EW}(\mathrm{H} \alpha)\right)}{\sum_{r<R} m_{*, i}} .
$$

Here $m_{*, i}$ is the stellar mass of the $i$ th spaxel derived with STARLIGHT, and $f_{i}$ determines whether the $i$ th spaxel is quenched or not. A spaxel is quenched if $\mathrm{D}_{n}(4000)>1.6$ and $\mathrm{EW}(\mathrm{H} \alpha)<2.0 \AA$ (Geha et al. 2012), so $f_{i}$ can be written as

$$
f_{i}= \begin{cases}1, & \mathrm{D}_{n}(4000)_{i}>1.6 \text { and } \mathrm{EW}(\mathrm{H} \alpha)_{i}<2.0 \\ 0, & \text { Otherwise }\end{cases}
$$

where $\mathrm{D}_{n}(4000)_{i}$ and $\mathrm{EW}(\mathrm{H} \alpha)_{i}$ are the $4000 \AA$ break and $\mathrm{H} \alpha$ emission-line EW for the $i$ th spaxel. When calculating $f_{Q}(R)$, 


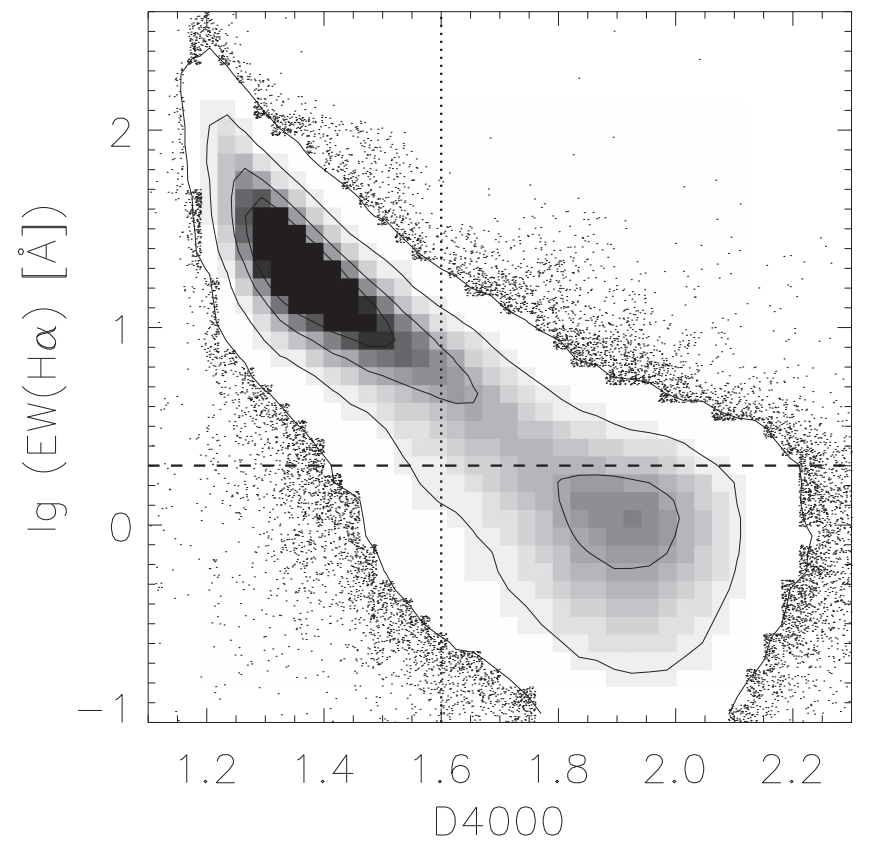

Figure 3. Distribution of all the individual spaxels in our galaxies on the plane of $\mathrm{D}_{n}(4000)$ vs. $\log _{10} \mathrm{EW}(\mathrm{H} \alpha)$. The horizontal dashed line and the vertical dotted line indicate $\mathrm{EW}(\mathrm{H} \alpha)=2 \AA$ and $\mathrm{D}_{n}(4000)=1.6$, respectively. The gray scale represents the number density of spaxels in linear space on this diagram.

we have corrected the inclination effect for each galaxy based on the minor-to-major axis ratio taken from the NSA.

In this definition, we have used both $\mathrm{D}_{n}(4000)$ and $\mathrm{EW}(\mathrm{H} \alpha)$ to identify quenched spaxels. Figure 3 displays the distribution of all the spaxels of the sample galaxies on the plane of $\mathrm{D}_{n}(4000)$ and $\log _{10} \mathrm{EW}(\mathrm{H} \alpha)$. The horizontal dashed line is for $\mathrm{EW}(\mathrm{H} \alpha)=2 \AA$, and the vertical dotted line is for $\mathrm{D}_{n}(4000)=1.6$. The spaxels are roughly separated into two regions: SF regions with a higher $\mathrm{EW}(\mathrm{H} \alpha)$ and lower $\mathrm{D}_{n}(4000)$ and quenched regions with a lower $\mathrm{EW}(\mathrm{H} \alpha)$ and higher $\mathrm{D}_{n}(4000)$. This is in agreement with the bimodality of the stellar population on kpc scales (Zibetti et al. 2017). As can be seen, the spaxels with $D_{n}(4000)>$ 1.6 are mostly but not always below the line of $\operatorname{EW}(\mathrm{H} \alpha)=2 \AA$. A significant fraction of the spaxels with $\mathrm{D}_{n}(4000)>1.6$ can have an $\mathrm{EW}(\mathrm{H} \alpha)$ as high as $\sim 10 \AA$. In previous studies, regions with $\mathrm{EW}(\mathrm{H} \alpha)>6 \AA$ were usually classified as SF regions (e.g., Sánchez et al. 2014), while regions with $\mathrm{EW}(\mathrm{H} \alpha)<6 \AA$ appeared to be diffuse ionized gas, likely ionized by post-asymptotic giant branch (AGB) stars (e.g., Papaderos et al. 2013; Belfiore et al. 2017; Zhang et al. 2017). The criterion of $\operatorname{EW}(\mathrm{H} \alpha)<2 \AA$, as an addition to the requirement of $\mathrm{D}_{n}(4000)>1.6$, is probably somewhat conservative but safe for identifying quenched regions (Geha et al. 2012).

In this paper, we adopt $R=1.5 \mathrm{R}_{\mathrm{e}}$ when measuring $f_{Q}$, considering that $1.5 \mathrm{R}_{\mathrm{e}}$ is the radius to which both the primary and secondary samples are observed with sufficient $S / N$. In Figure 4 , we show the histogram of $f_{Q}\left(1.5 \mathrm{R}_{\mathrm{e}}\right)$ of our sample (left panel) and the correlation of $f_{Q}\left(1.5 \mathrm{R}_{\mathrm{e}}\right)$ with both the global NUV $-r$ (middle panel) and the central $\mathrm{D}_{n}(4000)$ (right panel). It is striking that the majority of galaxies can be divided into two extreme cases with either $f_{Q}<0.1$ or $f_{Q}>0.9$, with only a small fraction falling in between. Therefore, we classify them into three subsets: SF galaxies with $f_{Q}\left(1.5 \mathrm{R}_{\mathrm{e}}\right)<0.1$, PQ galaxies with $0.1 \leqslant f_{Q}\left(1.5 \mathrm{R}_{\mathrm{e}}\right)<0.9$, and TQ galaxies with $f_{Q}\left(1.5 \mathrm{R}_{\mathrm{e}}\right) \geqslant 0.9$. As a result, our sample includes $993 \mathrm{SF}$ galaxies, $347 \mathrm{PQ}$ galaxies, and 577 TQ galaxies. Having performed the $1 / V_{\max }$ correction, we find that the $\mathrm{SF}$ population dominates the sample $(\sim 69.5 \%)$, TQ galaxies account for $18.4 \%$ of the total, and only $\sim 12.1 \%$ are in the class of PQ.

Galaxies in the blue cloud with NUV $-r<4$ and those with central $\mathrm{D}_{n}(4000)<1.6$ are mostly classified as $\mathrm{SF}$ galaxies with $f_{Q}\left(1.5 \mathrm{R}_{\mathrm{e}}\right)<0.1$; that is, more than $90 \%$ of the area of these galaxies is forming stars. On the other hand, SF galaxies are not always blue or CSF. Rather, the SF galaxies may have redder colors with NUV $-r>4$ or quenched centers with $\mathrm{D}_{n}(4000)>1.6$. This implies that the central region of these galaxies is quenched while the outskirts are still forming stars. Galaxies with NUV $-r>4$ or central $\mathrm{D}_{n}(4000)>1.6$ span a full range of $f_{Q}\left(1.5 \mathrm{R}_{\mathrm{e}}\right)$, suggesting that the global color or central spectroscopy cannot accurately reflect the overall status of the star formation in these galaxies.

In Figure 5, we show the color-mass and BPT diagrams for the sample galaxies again, using the same SDSS-based parameters as in Figure 1 but plotting the SF/PQ/TQ galaxies in blue/green/red symbols, respectively. The SF and TQ galaxies are well separated in the NUV $-r$ versus $M_{*}$ diagram, while the PQ galaxies are broadly distributed over a wide color range above NUV $-r \sim 3$. The TQ galaxies are mostly redder than NUV $-r=5$, which is the commonly adopted cut for selecting red-sequence galaxies. ${ }^{18}$ This demonstrates that the red global color is a necessary but insufficient criterion for identifying a fully quenched galaxy. Similarly, blue-cloud galaxies, as usually selected by NUV $-r<4$, are mostly but not purely SF galaxies. The green valley enclosed by the red and blue divisions, i.e., $4<\mathrm{NUV}-r<5$, is a mixture of the SF and PQ galaxies with very few TQ galaxies. Interestingly, the relative fractions of the SF and PQ classes in the green valley depend on the stellar mass, in the sense that the SF population dominates at low masses (below a few $\times 10^{10} M_{\odot}$ ) and the PQ population dominates at high masses.

In the BPT diagram, we find the SF galaxies to be distributed over all the different regions, although the majority reside in the expected SF region. A small but significant fraction of the SF galaxies are found in the composition and AGN area. On the other hand, the CSF galaxies, located below the dividing line of Kewley et al. (2006) in the BPT diagram, are mostly classified as fully SF galaxies according to the MaNGA data. This echoes the finding of Paper I, where CSF galaxies present weak or no gradients in the diagnostics of recent SFHs. Furthermore, we find that the PQ and TQ galaxies are similarly located in the composition and AGN regions, with the majority falling in the region of low-ionization nuclear emission-line region (LINER), or low-ionization emission-line region (LIER) (e.g., Belfiore et al. 2015, 2016; Zhang et al. 2017). This may suggest that the two classes of galaxies share similar ionizing sources in the nuclear region.

\subsection{Radial Profiles and Gradients of the SFH Diagnostics}

For each galaxy in our sample, we have obtained the radial profiles of the three diagnostic parameters: $\mathrm{D}_{n}(4000)$, $\operatorname{EW}\left(\mathrm{H} \delta_{A}\right)$, and $\operatorname{EW}(\mathrm{H} \alpha)$. We take the spaxels with a continuum $\mathrm{S} / \mathrm{N}>5$ at $5500 \AA$ in the two-dimensional map of a given parameter and divide them into a set of nonoverlapping radial

\footnotetext{
${ }^{18}$ We note that some authors have adopted a mass-dependent color division. As can be well expected from Figure 5, our conclusion that the TQ galaxies are mostly located in the red sequence would not change if a mass-dependent division was used.
} 

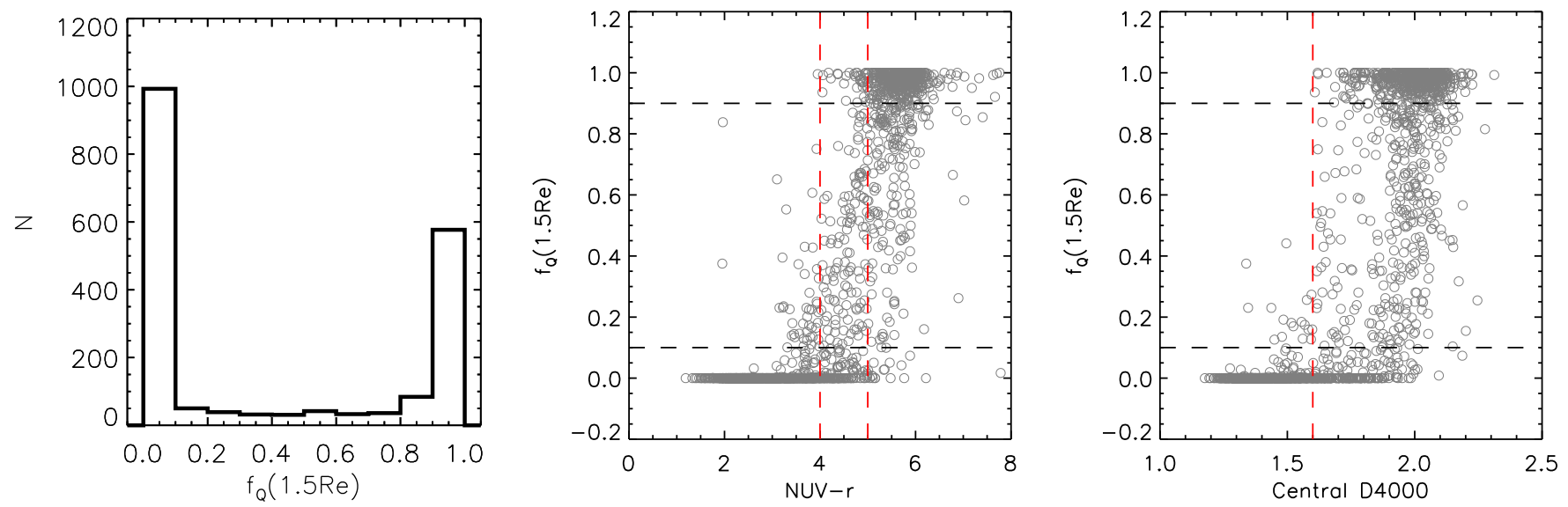

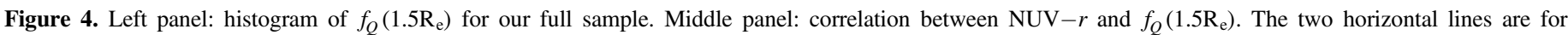

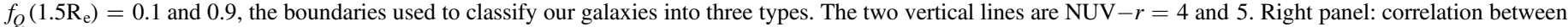
central $\mathrm{D}_{n}(4000)$ and $f_{Q}\left(1.5 \mathrm{R}_{\mathrm{e}}\right)$. The two horizontal lines are for $f_{Q}\left(1.5 \mathrm{R}_{\mathrm{e}}\right)=0.1$ and 0.9 , while the vertical line indicates that $\mathrm{D}_{n}(4000)=1.6$.

bins with a constant interval of $\Delta\left(R / \mathrm{R}_{\mathrm{e}}\right)=0.2$. The radial profile of the parameter is then derived from the median value of the spaxels falling in each radial bin. While doing this, we have corrected the inclination effect for each spaxel based on the minor-to-major axis ratio from the NSA.

Figure 6 displays the radial profiles of one of the three diagnostic parameters, $\mathrm{D}_{n}(4000)$, for all of the galaxies in our sample. We divide the galaxies into five stellar mass intervals: $\log _{10}\left(M_{*} / M_{\odot}\right)<9.8, \quad 9.8 \leqslant \log _{10}\left(M_{*} / M_{\odot}\right)<10.2, \quad 10.2 \leqslant$ $\log _{10}\left(M_{*} / M_{\odot}\right)<10.6, \quad 10.6 \leqslant \log _{10}\left(M_{*} / M_{\odot}\right)<11.0$, and $11.0 \leqslant \log _{10}\left(M_{*} / M_{\odot}\right)$. For each stellar mass interval, we present the results for the SF, PQ, and TQ galaxies separately. In the figure, the panels from left to right correspond to the different mass bins, while the panels from top to bottom correspond to the three different galaxy types. In each panel, we show the median radial profile of $\mathrm{D}_{n}(4000)$ as black dots connected by a solid line, as well as the $1 \sigma$ scatter around the median profile as error bars. When estimating the median profiles, we have corrected the selection effect of the MaNGA target sample by weighting each galaxy by $1 / V_{\max }$ as described in the previous section. The profiles are plotted as a function of $R / \mathrm{R}_{\mathrm{e}}$, which is the galaxy-concentric radius scaled by the effective radius.

A number of interesting results can be seen in this figure. First, for a given galaxy type, both the amplitude and the slope of the $\mathrm{D}_{n}(4000)$ profile vary with stellar mass, with lower amplitudes and flat slopes at masses below $\sim 10^{10} M_{\odot}$ and higher amplitudes and steeper negative slopes at higher masses. The trend with mass is more pronounced for SF and PQ galaxies, and the effect is rather weak for TQ galaxies, which present no or very weak gradients in $\mathrm{D}_{n}(4000)$ at all masses. Second, at a fixed mass, the three types of galaxies present different profiles, but only at intermediate-to-high masses (above $\sim 10^{10.2} M_{\odot}$; hereafter, we refer to galaxies with a stellar mass above $\sim 10^{10.2}$ as massive galaxies and those below as less massive galaxies.). At these masses, SF and PQ galaxies show similarly steep profiles with smaller $\mathrm{D}_{n}(4000)$ at larger radii, while TQ galaxies present relatively large $\mathrm{D}_{n}(4000)$ over all the radii with no/weak radial variations. At lower masses, different types of galaxies show similarly flat profiles but different amplitudes, with $\mathrm{D}_{n}(4000)$ averaged at $\sim 1.4, \sim 1.6$, and $\sim 1.8$ for SF, PQ, and TQ galaxies, respectively.
In most cases, the radial profile of $\mathrm{D}_{n}(4000)$ within the effective radius can be simply described by a straight line, consistent with the finding of Paper I. Therefore, following Paper I, we have performed a linear fit to the $\mathrm{D}_{n}(4000)$ profile at $R<\mathrm{R}_{\mathrm{e}}$ for each galaxy. Figure 7 presents the distributions of the best-fit slope, $\alpha\left(\mathrm{D}_{n}(4000)\right)$, which is the change in $\mathrm{D}_{n}(4000)$ per $\mathrm{R}_{\mathrm{e}}$, for the $\mathrm{SF}$ (blue lines), $\mathrm{PQ}$ (green lines), and $\mathrm{TQ}$ (red lines) galaxies in the same five mass ranges. In each panel, we perform a Kolmogorov-Smirnov (K-S) test to quantify the significance of the statistical differences between every two distributions, as indicated in the top right corner of each panel. An impressive result from this figure is the similarity between the SF and the PQ galaxies, which show very similar distributions of $\alpha\left(\mathrm{D}_{n}(4000)\right)$ at a fixed mass. In addition, both the no/weak gradients of all diagnostics in the less massive galaxies and the mass dependence in the massive galaxies can be easily identified from the figure. For the less massive galaxies, the distribution of $\alpha\left(\mathrm{D}_{n}(4000)\right)$ is constant at around zero with a narrow width of $\sim 0.1$, and the distribution is pretty much the same for the three types of galaxies. For the massive galaxies, the SF and PQ galaxies still show similar distributions but are centered at more negative $\alpha\left(\mathrm{D}_{n}(4000)\right)$ values and with larger widths when compared to the distributions of the less massive galaxies. The $\mathrm{D}_{n}(4000)$ gradient in the TQ galaxies is less dependent on stellar mass, with similarly narrow widths at all masses and slightly stronger (negative) gradients at higher masses.

Figure 8 presents the normalized median profiles of all three diagnostic parameters separately for the five stellar mass intervals. For each galaxy in our sample, we normalize the radial profiles by subtracting the values at $0.5 \mathrm{R}_{\mathrm{e}}$, and we obtain the median profile for the given stellar mass range and galaxy type in the same way as above. The trends of the $\mathrm{D}_{n}(4000)$ gradient with both the stellar mass and star formation status as seen in the previous figure are more clearly seen in the top panels of the current figure. Furthermore, as shown in the middle and bottom panels, the other two diagnostic parameters, $\mathrm{EW}\left(\mathrm{H} \delta_{A}\right)$ and $\mathrm{EW}(\mathrm{H} \alpha)$, show quite consistent behaviors with $\mathrm{D}_{n}(4000)$ in terms of radial gradients. The less massive galaxies with $M_{*} \lesssim 10^{10.2} M_{\odot}$ show weak gradients regardless of the overall star formation status. For the massive galaxies, the gradients of the diagnostic parameters depend on both the stellar mass and the star formation status. The TQ galaxies 

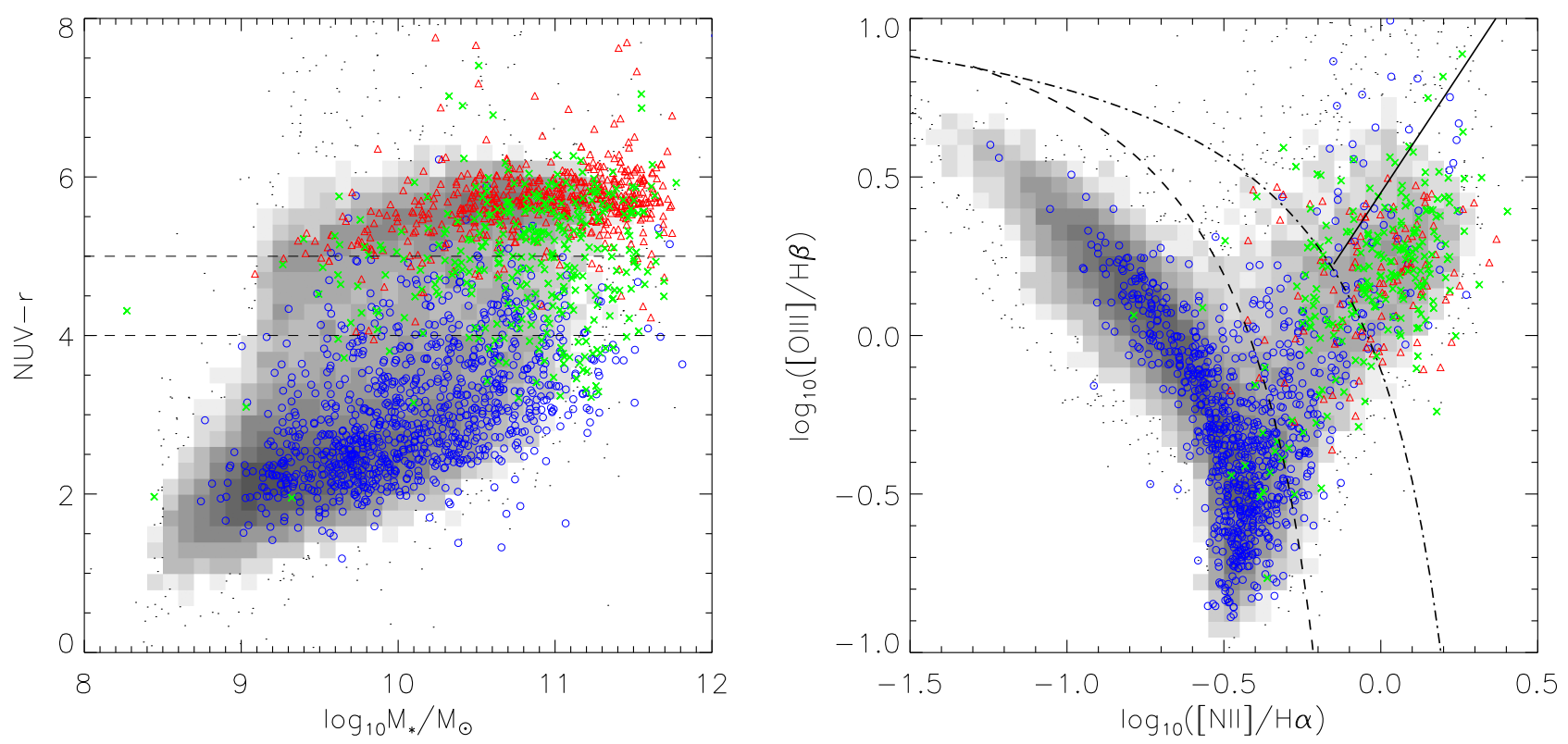

Figure 5. Galaxies are shown on the stellar mass vs. NUV $-r$ plane (left panel) and the BPT (Baldwin et al. 1981) diagram (right panel). The figure is similar to Figure 1, but the colors of the symbols reflect the overall star formation status of the galaxies, which is fully SF (blue), PQ (green), or TQ (red).

show almost no gradients in $\mathrm{EW}(\mathrm{H} \alpha)$ and similarly weak gradients in $\mathrm{D}_{n}(4000)$ and $\mathrm{EW}\left(\mathrm{H} \delta_{A}\right)$, while the $\mathrm{SF}$ and PQ galaxies show similar positive gradients in all the diagnostics with stronger gradients at higher masses.

One may worry that the gradients of our diagnostic parameters might be affected by the beam-smearing effect due to the limited spatial resolution of the MaNGA IFUs. In fact, the great majority of the galaxies in the MaNGA primary and secondary samples are well resolved (Ibarra-Medel et al. 2016). About $92 \%$ of the sample galaxies have an $R_{e}$ larger than 2."5, the effective spatial resolution of the MaNGA data cubes, and more than $97 \%$ have a $1.5 \mathrm{R}_{\mathrm{e}}$ larger than $2 . " 5$. We have also examined the potential dependence of $\mathrm{D}_{n}(4000)$ gradients on galaxy angular size for the primary and secondary samples separately, finding no significant trends. Therefore, the beam-smearing effect should not have a significant impact on the results.

\subsection{Diagnostic Diagrams of Recent SFHs}

In the previous subsection, we examined the radial profiles of the three diagnostics. In this subsection, we jointly analyze the different diagnostics by showing their radial profiles on three diagrams of $\mathrm{D}_{n}(4000)$ versus $\mathrm{EW}\left(\mathrm{H} \delta_{A}\right), \mathrm{EW}\left(\mathrm{H} \delta_{A}\right)$ versus $\mathrm{EW}(\mathrm{H} \alpha)$, and $\mathrm{EW}(\mathrm{H} \alpha)$ versus $\mathrm{D}_{n}(4000)$. To this end, we take the data points of each radial profile at four different radii: 0.1, $0.5,0.9$, and $1.3 \mathrm{R}_{\mathrm{e}}$. For each radius, we then show the distribution of the galaxies on the three diagnostic diagrams. The results are shown in Figures 9-11. The panels from left to right correspond to the five stellar mass ranges, while the panels from top to bottom correspond to the four radii. Thus, each panel displays a diagram for a given stellar mass bin in a given radial bin. In each panel, the blue circles, green crosses, and red triangles represent the SF, PQ, and TQ galaxies falling in the stellar mass range. For comparison, we plot all the spaxels of galaxies in the corresponding stellar mass range that have a continuum SNR $>5$ at $5500 \AA$ as the background grayscale distribution in each panel. The $\mathrm{BC} 03$ models with continuously declining star formation and bursting star formation are plotted as the solid and dashed lines. We note that we have adopted $\mathrm{BC} 03$ models of different metallicities for different mass bins, considering the known metallicity dependence on mass (Lequeux et al. 1979; Garnett \& Shields 1987; Tremonti et al. 2004; Gallazzi et al. 2005; Panter et al. 2008; Sánchez et al. 2013). We use models of $Z=0.4 Z$. for the less massive galaxies in the two lowest stellar mass bins and models of $Z=Z_{\odot}$ for the massive galaxies in the other three mass bins.

A general result of these figures is that the distributions of galaxies on these diagrams broadly follow the continuous star formation models, although a small fraction of individual spaxels may extend to regions of starbursts. This is true for all masses and radii and independent of the overall star formation status of the galaxies. A galaxy may be located differently in the diagrams, depending on mass, radius, and classification, but the location is confined to the regions of continuous star formation models. For instance, in Figure 9, we see that the PQ galaxies (green crosses) in the top right panel $\left(\log _{10}\left(M_{*} / M_{\odot}\right)>11, R=0.1 \mathrm{R}_{\mathrm{e}}\right)$ are mostly located in the quenched region with the largest $\mathrm{D}_{n}(4000)$ and smallest $\mathrm{EW}\left(\mathrm{H} \delta_{A}\right)$, and they are moving along the tight sequence of continuous star formation models to more $\mathrm{SF}$ regions with smaller $\mathrm{D}_{n}(4000)$ and larger $\mathrm{EW}\left(\mathrm{H} \delta_{A}\right)$ when one goes to larger radii. This result reflects the radial profile of PQ galaxies at fixed mass, as already presented in previous figures. However, the tight sequence on the $\mathrm{D}_{n}(4000)-\mathrm{EW}\left(\mathrm{H} \delta_{A}\right)$ diagram can only be clearly seen when the diagnostics are jointly examined. It is striking to see the same tight sequence on the diagnostic diagram holding regardless of mass, radius, and galaxy type. But one should keep in mind that we have excluded mergers and irregular and disturbed galaxies from our sample. The result suggests that, as pointed out in Paper I, the star formation cessation in a galaxy must be a smooth long-term process, likely governed by a common set of drivers, and starburst activities happen rarely in galaxies with regular morphologies. We emphasize that the lack of starburst spaxels is not due to problematic spectral fitting of post-starburst galaxies. We have 


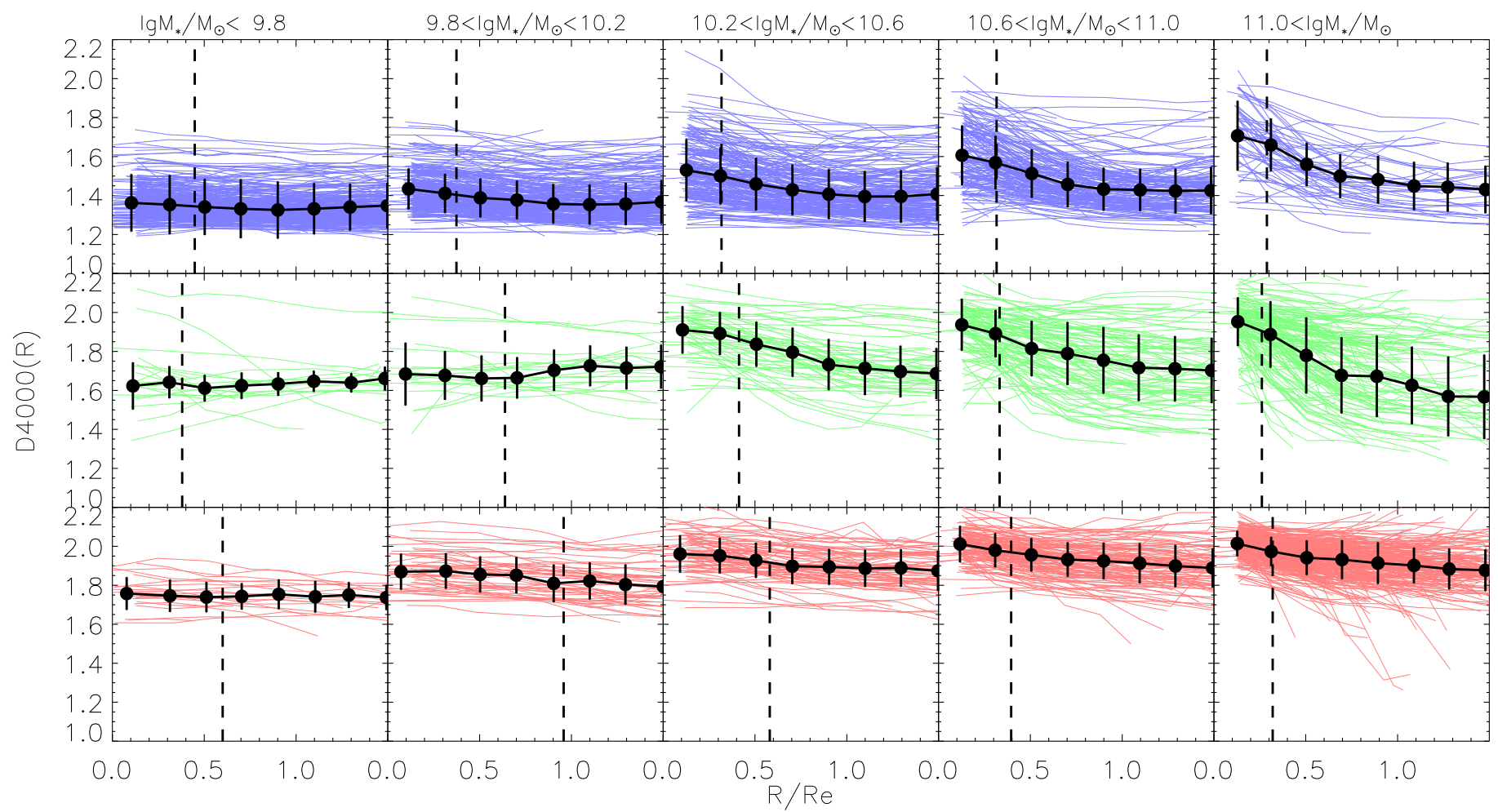

Figure 6. Radial profiles of $\mathrm{D}_{n}(4000)$ plotted as a function of radius scaled by effective radius. The panels from left to right are the results for five different stellar mass intervals, as indicated. The panels from top to bottom present results for the three types of galaxies separately: fully SF galaxies, PQ galaxies, and TQ galaxies. The thin colored lines are for individual galaxies. In each panel, the dots connected with the solid line plot the median profile of all of the galaxies in the panel, and error bars indicate the $1 \sigma$ scatter of individual galaxies around the median relation. In each panel, the vertical dashed line shows the typical resolution of each subsample.

examined some known post-starburst galaxies in our sample, finding their spectra to be reasonably well fitted.

The distributions of the galaxies on the other two diagrams, both involving $\mathrm{EW}(\mathrm{H} \alpha)$, are not as tight as those in the $\mathrm{D}_{n}(4000)$ versus $\mathrm{EW}\left(\mathrm{H} \delta_{A}\right)$ diagram. However, even with a more scattered distribution, the galaxies are still barely found in the regions of starburst models, reinforcing the conclusion that the SFH in individual spaxels is consistent with the continuous star formation model. Furthermore, we find that the PQ and TQ classes are better separated on the $\mathrm{EW}(\mathrm{H} \alpha)$-related diagrams, with TQ galaxies located mostly below $\log _{10} \mathrm{EW}(\mathrm{H} \alpha) \sim 0$ and PQ galaxies above it.

We note that there are outliers (though small in number) in some panels, especially the panels for $R=0.1 \mathrm{R}_{\mathrm{e}}$ and highmass bins in Figures 9 and 10. It is interesting that the outliers in these panels are no longer discrepant when one moves to larger radii, implying that they are mostly caused by the inner regions of massive galaxies. We have visually inspected the spectra of the outliers, finding their emission lines to present a very broad component, thus indicating the presence of a Type I AGN. Special treatments and analyses of AGNs are beyond the scope of this paper, and the interested reader is referred to Belfiore et al. (2016) and Zhang et al. (2017) for detailed studies of AGNs and diffuse ionized gas emission in MaNGA galaxies.

\subsection{The Central $D_{n}(4000)$ versus $\alpha\left(D_{n}(4000)\right)$ Relation}

In the previous section, we examined the recent SFHs for individual spaxels at different radii within the galaxies, finding them to uniformly follow the BC03 models with continuously declining SFR, a result that is interestingly independent of stellar mass, radius, and the overall star formation status of galaxies. In this subsection, we make direct comparisons between the central and outer regions of the galaxies in order to understand whether there is any evolution relation across the galaxy. We concentrate on one of the three diagnostics, $\mathrm{D}_{n}(4000)$, for simplicity. Figure 12 compares the $\mathrm{D}_{n}(4000)$ measured in the central spaxel $\left(\mathrm{D}_{n}(4000)_{\text {cen }}\right)$ with both the $\mathrm{D}_{n}(4000)$ gradient $\left(\alpha\left(\mathrm{D}_{n}(4000)\right)\right.$, left panel) and the $\mathrm{D}_{n}(4000)$ measured at $1.5 \mathrm{R}_{\mathrm{e}}\left(\mathrm{D}_{n}(4000)_{1.5 \mathrm{Re}}\right.$, right panel). In both panels, galaxies are divided into SF (blue circles), PQ (green crosses), and TQ (red triangles) populations.

As can be seen, the SF and TQ populations can be well separated in both panels, while PQ galaxies are largely found in between, with substantial overlap with the TQ population. Consistent with the radial profiles shown in previous figures, the majority of the SF galaxies have the lowest $\mathrm{D}_{n}(4000)$ in the center, as well as weak/no gradients. Therefore, they are located in the upper left part of the $\mathrm{D}_{n}(4000)_{\text {cen }}$ versus $\alpha\left(\mathrm{D}_{n}(4000)\right)$ plane and the lower left part of the $\mathrm{D}_{n}(4000)_{\text {cen }}$ versus $\mathrm{D}_{n}(4000)_{1.5 \mathrm{Re}}$ plane, where $\mathrm{D}_{n}(4000)_{\text {cen }}<1.6$, $\alpha\left(\mathrm{D}_{n}(4000)\right)>-0.2$, and $\mathrm{D}_{n}(4000)_{1.5 \mathrm{Re}}<1.6$. In addition, it is interesting to see that a significant fraction of SF galaxies present strong gradients and high $\mathrm{D}_{n}(4000)$ in their centers $\left(\mathrm{D}_{n}(4000)>1.6\right)$ but low $\mathrm{D}_{n}(4000)$ at $1.5 \mathrm{R}_{\mathrm{e}}$. They are classified as SF because most of their spaxels fulfill the criteria for SF regions, and only the central region is quenched. As a whole, the SF galaxies form a nearly linear sequence on the $\mathrm{D}_{n}(4000)_{\text {cen }}$ versus $\alpha\left(\mathrm{D}_{n}(4000)\right)$ plane. The TQ galaxies are mostly located in the top right corner in both panels. They span a relatively narrow range in $\mathrm{D}_{n}(4000)$ at both the galactic center and $1.5 \mathrm{R}_{\mathrm{e}}$, with $\mathrm{D}_{n}(4000)>1.8$ and $\alpha\left(\mathrm{D}_{n}(4000)\right)>-0.5$ in most cases. The distribution of PQ galaxies is more scattering 


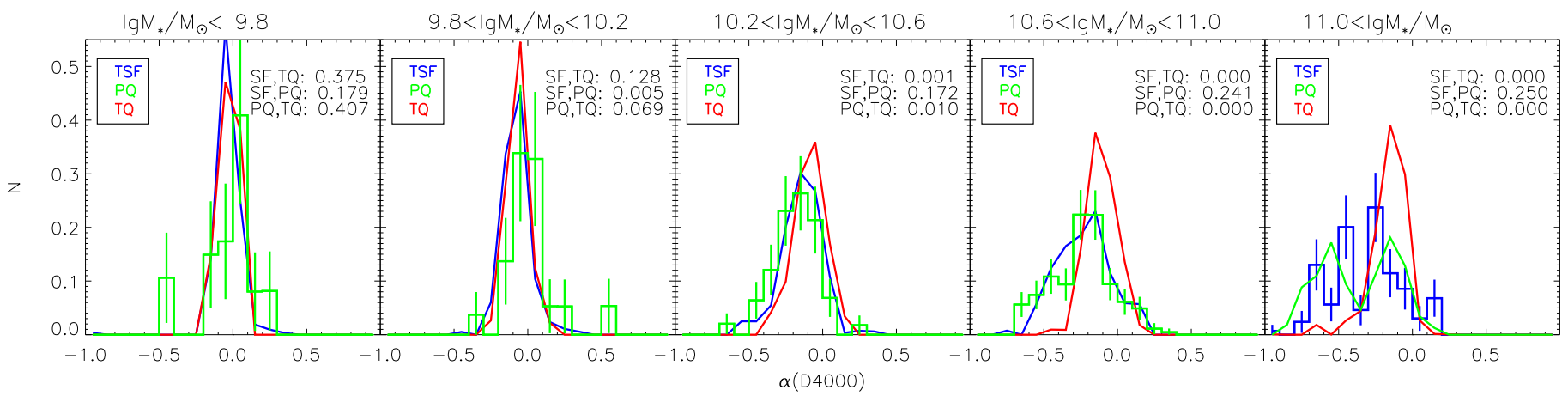

Figure 7. Histograms of the $\mathrm{D}_{n}(4000)$ slope index, $\alpha(D 4000)$, for galaxies with different stellar mass (panels from left to right) and at different star formation status, with blue/green/red lines for the SF, PQ, and TQ galaxies, respectively. The K-S probabilities of the distributions of every two subsamples are indicated in the top right corner of each panel. In each stellar mass bin, we show the Poisson errors for the subsample with the least number of galaxies.

when compared to the other two classes, covering both the intermediate region between the SF and TQ populations and the whole region of the TQ population but with little overlap with the SF population.

These results are in good agreement with the "inside-out" picture of star formation cessation, in which star formation gets shut down in the galactic center before propagating to larger and larger radii. In this picture, a fully SF galaxy starting with a flat $\mathrm{D}_{n}(4000)$ distribution with $\mathrm{D}_{n}(4000)$ constant at the lowest values $\left(\mathrm{D}_{n}(4000) \sim 1.2\right)$ will have larger $\mathrm{D}_{n}(4000)_{\text {cen }}$ and steeper slopes as star formation cessation happens from the center outward. Initially located in the upper left corner in the $\mathrm{D}_{n}(4000)_{\text {cen }}$ versus $\alpha\left(\mathrm{D}_{n}(4000)\right)$ plane, the galaxy appears to move along the SF sequence toward the lower right corner, where it deviates from the SF sequence and moves upward with central $\mathrm{D}_{n}(4000)$ saturated at $\mathrm{D}_{n}(4000)_{\text {cen }} \sim 2$ and $\alpha\left(\mathrm{D}_{n}(4000)\right)$ increasing from $\sim-0.8$ (steepest slopes) to around zero (flat slopes). The same picture can also be seen in the right panel, where the galaxy starts from the lower left corner with the same $\mathrm{D}_{n}(4000)$ at $R=0$ and $1.5 \mathrm{R}_{\mathrm{e}}$; evolves to the PQ phase, which shows the largest deviation from the 1:1 relation because of the strongest gradients in $\mathrm{D}_{n}(4000)$; and comes back to the $1: 1$ relation when the galaxy gets TQ, thus showing the highest $\mathrm{D}_{n}(4000)$ in both the center and the outskirts. The PQ galaxies are in the transition phase between the SF and TQ populations, given their locations on both diagrams.

Although the two panels generally illustrate the "inside-out" star formation cessation process, it is apparent that some individual galaxies do not simply follow this sequence. For instance, some SF galaxies appear to deviate from the SF sequence with a rather flat profile, falling between the SF sequence and the region of TQ galaxies in the left panel. In the right panel, these galaxies follow the 1:1 relation even when their central $\mathrm{D}_{n}(4000)$ exceeds 1.6. These galaxies are obviously not following the "inside-out" picture. In order to understand what drives the diversity in these panels, we have examined the possible dependence on stellar mass, morphology, and structural parameters. The results are shown in Figures 13-15.

In Figure 13, we repeat the two panels of the previous figure but for the five stellar mass bins separately. An immediate result from this figure is that the "inside-out" process as described above is seen only for massive galaxies in the three high-mass samples with masses above $10^{10.2} M_{\odot}$, with the trend becoming stronger at higher masses. In these mass bins, the galaxies are clearly offset from the one-to-one line, with the deviation increasing gradually with increasing stellar mass. For less massive galaxies, we see no/weak signature of "inside-out" in the sense that the galaxies show fairly flat $\mathrm{D}_{n}(4000)$ profiles, although the central $\mathrm{D}_{n}(4000)$ spans a full range of $1.2<\mathrm{D}_{n}(4000)<2$. We note that there are also a small number of massive SF galaxies with $\mathrm{D}_{n}(4000)_{\text {cen }}<\mathrm{D}_{n}(4000)_{1.5 \mathrm{Re}}$, suggesting that they have a different star formation cessation picture from that of normal massive SF galaxies. These galaxies have slightly positive gradients in $\mathrm{D}_{n}(4000)$ and negative gradients in $\log _{10} \mathrm{EW}(\mathrm{H} \alpha)$. Wang et al. (2017) used the public MaNGA data from SDSS DR13 and examined these galaxies, finding them to have a smaller size, higher concentration, higher SFR, and higher gas-phase metallicity when compared to normal SF galaxies of similar masses. In addition, their median surface mass density profile falls between the profiles of normal SF galaxies and quiescent galaxies with the same stellar mass distributions, indicating that these galaxies are likely in the transition phase from normal SF galaxies to quiescent galaxies, with rapid ongoing central stellar mass assembly probably related to bulge growth.

In Figures 14 and 15, we further examine the dependence of the diagnostic diagrams on both the bulge-to-total ratio $\left(f_{\mathrm{B} / \mathrm{T}}\right)$ and morphological type (spheroid-like versus disklike) but showing the results only for the plane of $\mathrm{D}_{n}(4000)_{\text {cen }}$ and $\mathrm{D}_{n}(4000)_{1.5 \mathrm{Re}}$ for simplicity. The bulge mass fractions are taken from Simard et al. (2011), obtained by performing photometric decomposition modeling on the SDSS $r$-band image. The morphological classification is done by visually examining the $r$-band image of the sample galaxies. We see quite similar results from both figures in the following three aspects. First, the radial variations in $\mathrm{D}_{n}(4000)$, as seen at $\log _{10}\left(M_{*} / M_{\odot}\right)>10.2$ in Figure 13, are dominated by disklike galaxies in the class of $\mathrm{SF}$ or $\mathrm{PQ}$, which have small $\mathrm{B} / \mathrm{T}$ luminosity ratios. We note that this trend might be driven by the lack of SF spheroid-like galaxies. However, given the small number of SF spheroid-like galaxies at fixed $M_{*}$, we are unable to reliably tell whether the distribution of SF disklike galaxies on the diagram differs significantly from that of SF spheroidlike galaxies. We will come back to this point in the future when larger samples are available. Second, the TQ galaxies are mostly spheroid-like and have large $\mathrm{B} / \mathrm{T}$ ratios, consistent with previous studies finding that a dense bulge is usually found in a quenched galaxy (e.g., Fang et al. 2013; Bluck et al. 2014). Third, these effects are stronger at higher stellar masses. Stellar mass is apparently a primary driver for the star formation cessation process in local galaxies. These results imply that the presence of a central massive object, such as a bulge, is unlikely to play a driving role in quenching. In fact, an 

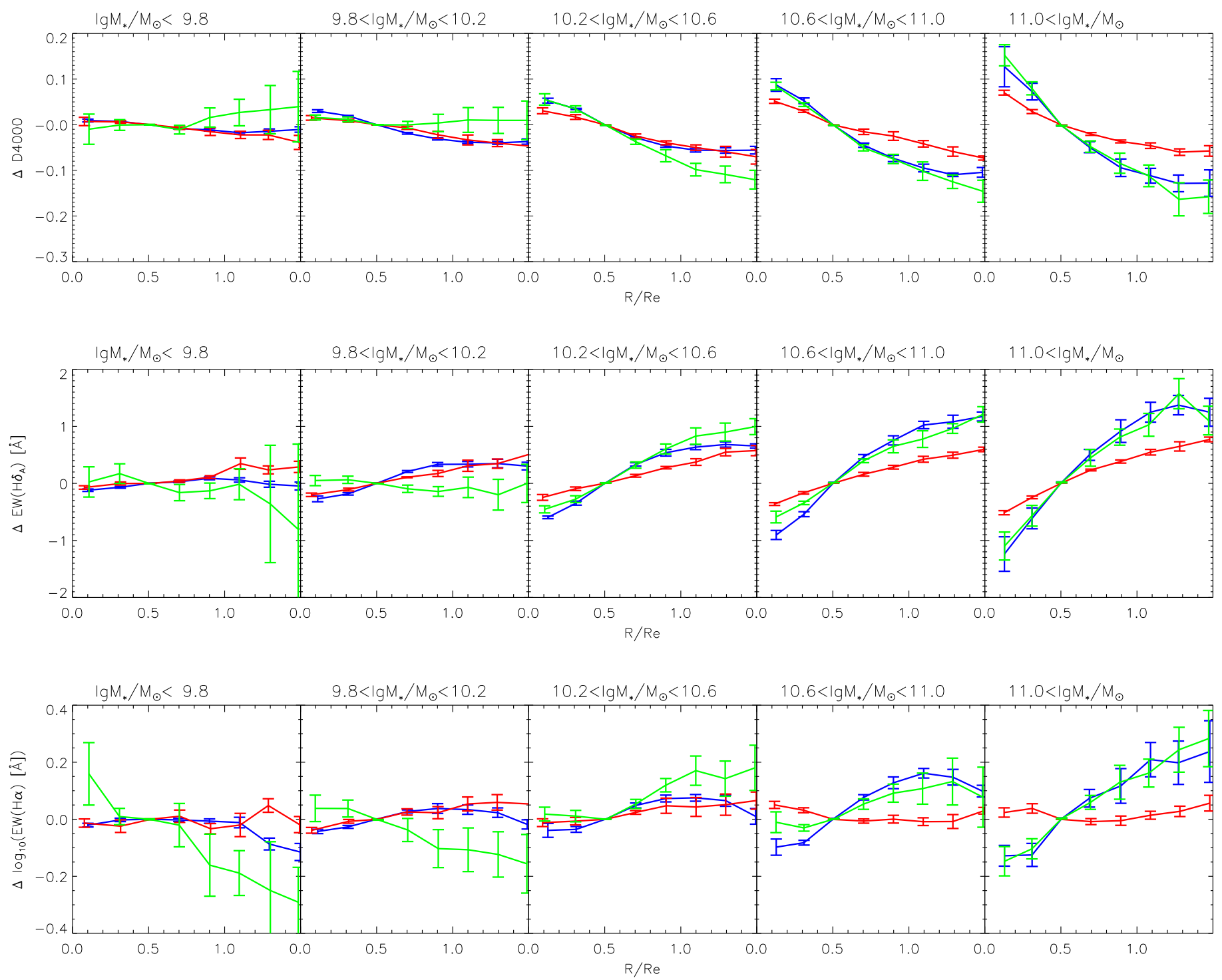

Figure 8. Median radial gradients in $\mathrm{D}_{n}(4000)$ (top panels), $\mathrm{EW}\left(\mathrm{H} \delta_{A}\right)$ (middle panels), and $\mathrm{EW}(\mathrm{H} \alpha)$ (bottom panels) for galaxies with different stellar masses (panels from left to right) and at different star formation statuses (red/green/blue corresponding to the SF, PQ, and TQ galaxies, respectively).

additional analysis shows that the significant deviation of the massive galaxies from the 1:1 relation, as seen in Figure 14, still holds even when we restrict ourselves to the galaxies with $f_{\mathrm{B} / \mathrm{T}}<0.2$. On the other hand, the absence of a bulge seems to be required in order to have a large radial gradient, which is an observational signature of the inside-out cessation process. However, this result should not be overemphasized due to the small number of SF galaxies with $f_{\mathrm{B} / \mathrm{T}}>0.4$. A spheroid-like morphology or large $f_{\mathrm{B} / \mathrm{T}}$ is associated mostly with galaxies at the TQ stage, implying that the growth of a central massive object is likely a consequence of star formation cessation and probably plays crucial roles only at the late stage of the quenching process.

\section{Discussion}

\subsection{Comparison with Previous Results}

Paper I investigated maps and radial profiles of $\mathrm{D}_{n}(4000)$, $\mathrm{EW}\left(\mathrm{H} \delta_{A}\right)$, and $\mathrm{EW}(\mathrm{H} \alpha)$ for a preliminary sample of 12 galaxies observed in P-MaNGA, finding that the individual spaxels of the P-MaNGA galaxies broadly follow models of continuously declining star formation and nicely form a tight sequence on the $\mathrm{D}_{n}(4000)-\mathrm{EW}\left(\mathrm{H} \delta_{A}\right)$ diagram. In this work, we have extended the analysis of Paper I by using a much larger sample of 1917 galaxies from the MaNGA MPL5 and find that those suggestive results are confirmed at much greater significance (see Figures 9-11). This indicates that the growth and death of galaxies must be a smooth process, and that starbursts happen rarely in galaxies with regular morphology.

Paper I classified galaxies as either CSF or CQ according to the star formation status of the central region and found that the two classes present distinct radial profiles in the diagnostic parameters. In this work, we found that the central region alone cannot accurately determine the overall star formation status of a galaxy. A galaxy with a quenched center could be forming stars in the outskirts, thus a PQ galaxy, or it could be a TQ galaxy with no star formation across the whole area. We have proposed a single classifier, $f_{Q}\left(R<1.5 \mathrm{R}_{\mathrm{e}}\right)$, based on the twodimensional map of both $\mathrm{D}_{n}(4000)$ and $\operatorname{EW}(\mathrm{H} \alpha)$ and made comparisons with conventional classifiers such as global NUV $-r$ color and central $\mathrm{D}_{n}(4000)$. 


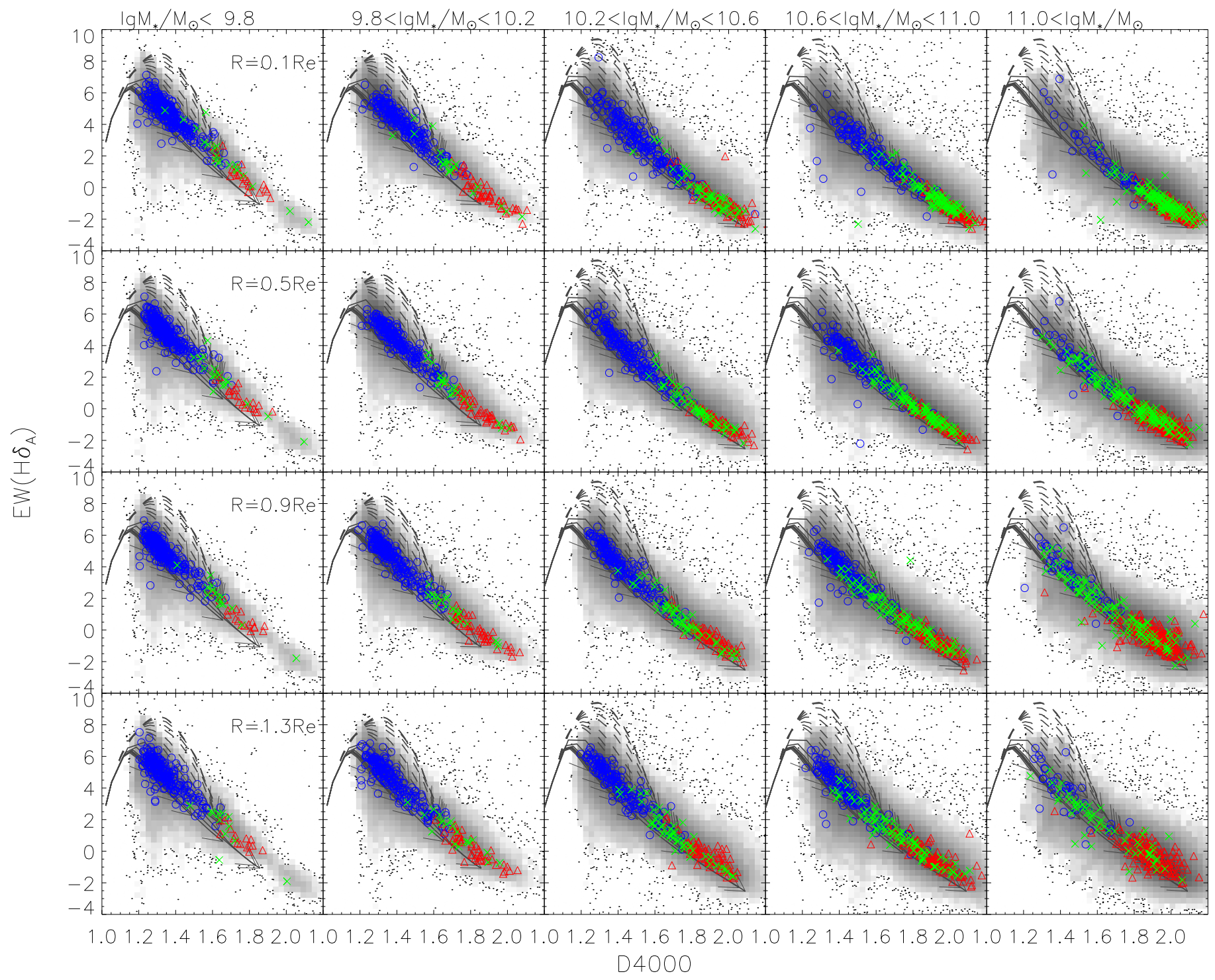

Figure 9. Distributions in the plane of the $\mathrm{D}_{n}(4000)$ and $\mathrm{H} \delta_{A}$ indices for different stellar mass intervals (panels from left to right) and radial bins (panels from top to bottom). The stellar mass ranges are indicated above the top panels, and the center radii of the radial bins are indicated in the leftmost panels. The blue/green/red symbols represent the spaxels from the corresponding mass and radial bins. The distribution of all spaxels from a given stellar mass bin is plotted as a gray-scale background for comparison. The lines in each panel are predictions of the BC03 models with either continuously declining star formation (solid lines) or starbursts (dashed lines). We adopt the BC03 models with $Z=0.4 Z_{\odot}$ for the lowest two stellar mass bins and those with $Z=Z_{\odot}$ for the other stellar mass bins. See the text for details.

Resolved stellar populations have been studied in recent years based on multiwavelength broadband photometry, longslit spectroscopy, and IFU spectroscopy for nearby galaxies (Pérez et al. 2013; González Delgado et al. 2014, 2015; Sánchez et al. 2014). Using 105 galaxies from CALIFA, Pérez et al. (2013) studied the spatially resolved history of their stellar mass assembly by applying the fossil record method, revealing an inside-out growth picture for massive galaxies and a transition to outside-in growth for low-mass galaxies $\left(\log _{10}\left(M_{*} / M_{\odot}\right)<10.0\right)$. Following this work, Ibarra-Medel et al. (2016) reconstructed the radial stellar mass growth histories of a large sample of galaxies from MaNGA. They also found an inside-out stellar mass growth picture for massive galaxies, and this picture is more pronounced in blue/SF/ late-type galaxies than in red/quiescent/early-type galaxies. In contrast, this picture does not hold for less massive galaxies with stellar mass below $\sim 10^{10} M_{\odot}$. In this work, we have concentrated our investigation on the star formation cessation process, rather than the growth process, by using the three diagnostics of recent SFH. We find that the inside-out star formation cessation picture is true only for massive galaxies. For less massive galaxies, we find the recent SFH to be fairly uniform across the galaxy, with no/weak radial gradients. Therefore, these new results support neither the simple insideout nor the outside-in picture for less massive galaxies.

Zheng et al. (2017) estimated the gradients in both stellar age and metallicity by applying the STARLIGHT software to an earlier sample from MaNGA, finding significantly negative gradients in age and weak gradients in metallicity. Using a similar MaNGA sample, Goddard et al. (2017) found negative gradients in stellar metallicity for late-type galaxies, with a stronger effect at higher masses. In this work, we have taken a less model-dependent approach by focusing our analysis on the three diagnostic parameters. For comparison, we present an analysis of stellar age and metallicity profiles in the Appendix, obtained by performing full spectral fitting to MaNGA data 


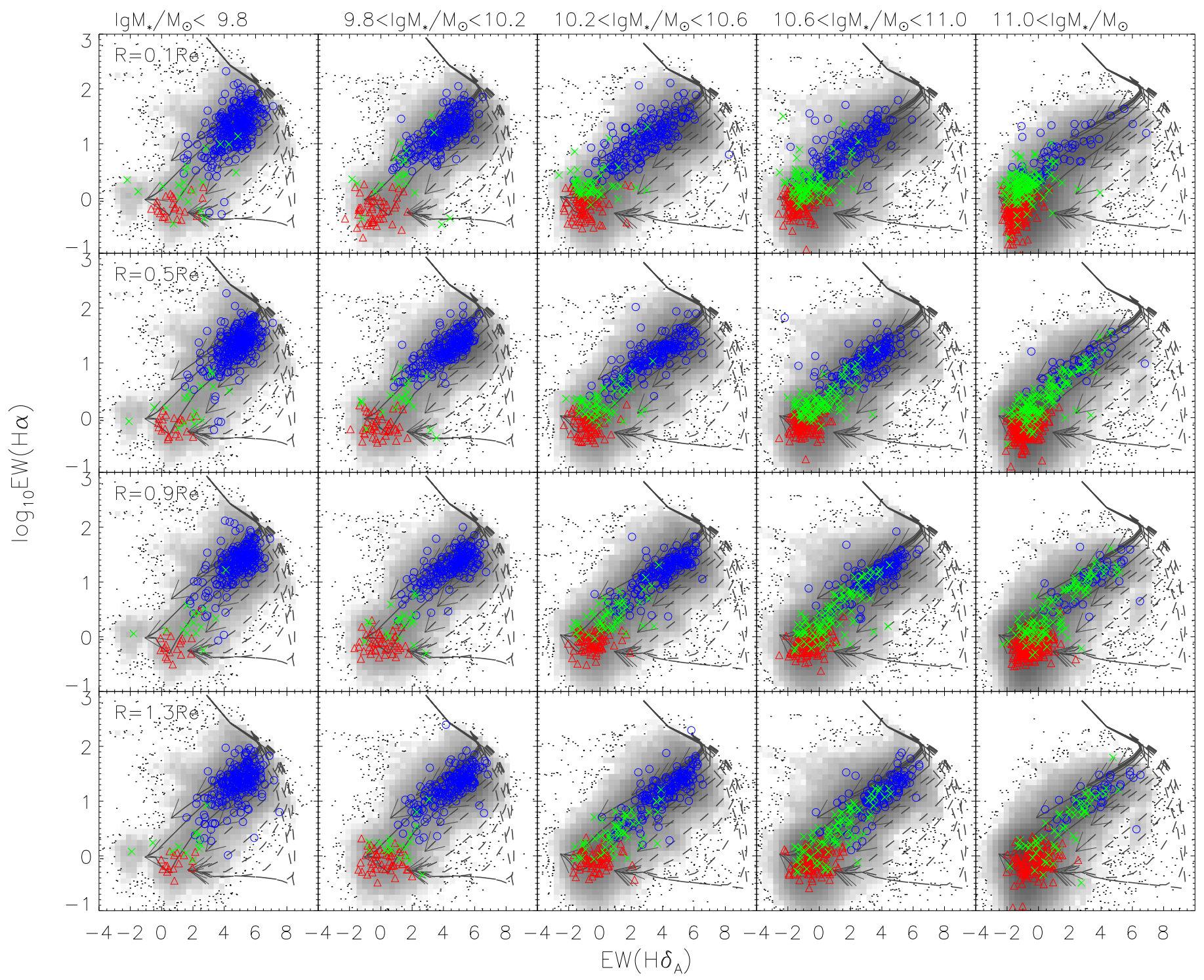

Figure 10. Same as Figure 9 but showing distributions in the plane of $\mathrm{EW}\left(\mathrm{H} \delta_{A}\right)$ and $\log _{10} \mathrm{EW}(\mathrm{H} \alpha)$.

cubes using STARLIGHT. Our results are consistent with what Zheng et al. (2017) found. The stellar age gradients obtained from STARLIGHT appear to be in good agreement with those in Goddard et al. (2017) as well. In contrast to Goddard et al. (2017), we have found rather weak gradients in stellar metallicity for massive SF and PQ galaxies. In a parallel MaNGA paper led by $\mathrm{H}$. Li et al. (2018, in preparation), we have done detailed comparisons between STARLIGHT and PPXF, finding them to be consistent with each other in terms of both stellar age and metallicity gradients. The discrepancy with Goddard et al. (2017) is probably due to the different spectral fitting procedures adopted in their code; more work is needed to ascertain the source of the discrepancy. In any case, the large gradients of $\mathrm{D}_{n}(4000)$, as shown for massive galaxies in our work, are consistent with the predictions of most of the commonly used models and so are unlikely to be caused by stellar metallicity gradients.

Kauffmann (2015) analyzed the recent SFH of low-mass galaxies from SDSS with stellar masses in the range $10^{8}-10^{10} M_{\odot}$, using $\mathrm{D}_{n}(4000)$ and $\mathrm{EW}\left(\mathrm{H} \delta_{A}\right)$ in combination with $\mathrm{SFR} / M_{*}$ as diagnostics. It was suggested that a large fraction of the SFR density in these galaxies was contributed by multiple starbursts triggered by gas cooling and supernova feedback cycles over the history of the galaxy. The current work does not attempt to derive any model-dependent SFH parameters such as $F_{\text {burst }}$, the fraction of stellar mass formed in bursts. We have analyzed both $\mathrm{D}_{n}(4000)$ and $\operatorname{EW}\left(\mathrm{H} \delta_{A}\right)$, and the third parameter, $\operatorname{EW}(\mathrm{H} \alpha)$, is expected to be correlated with SFR $/ M_{*}$. Therefore, our measurements of the radial profiles for these diagnostics should, in principle, provide useful constraints on the picture proposed by Kauffmann (2015), which was limited by the single-fiber spectroscopy from SDSS, thus probing the stellar populations only within the central $1-2 \mathrm{kpc}$ of a galaxy. Although the continuous star formation models of $\mathrm{BC} 33$ can explain the recent SFH for the majority of our galaxies in the same mass range, a model with multiple starbursts cannot simply be ruled out. It would be interesting to see whether their model and the continuous star formation models can be discriminated using the resolved spectroscopy from MaNGA in a future work. 


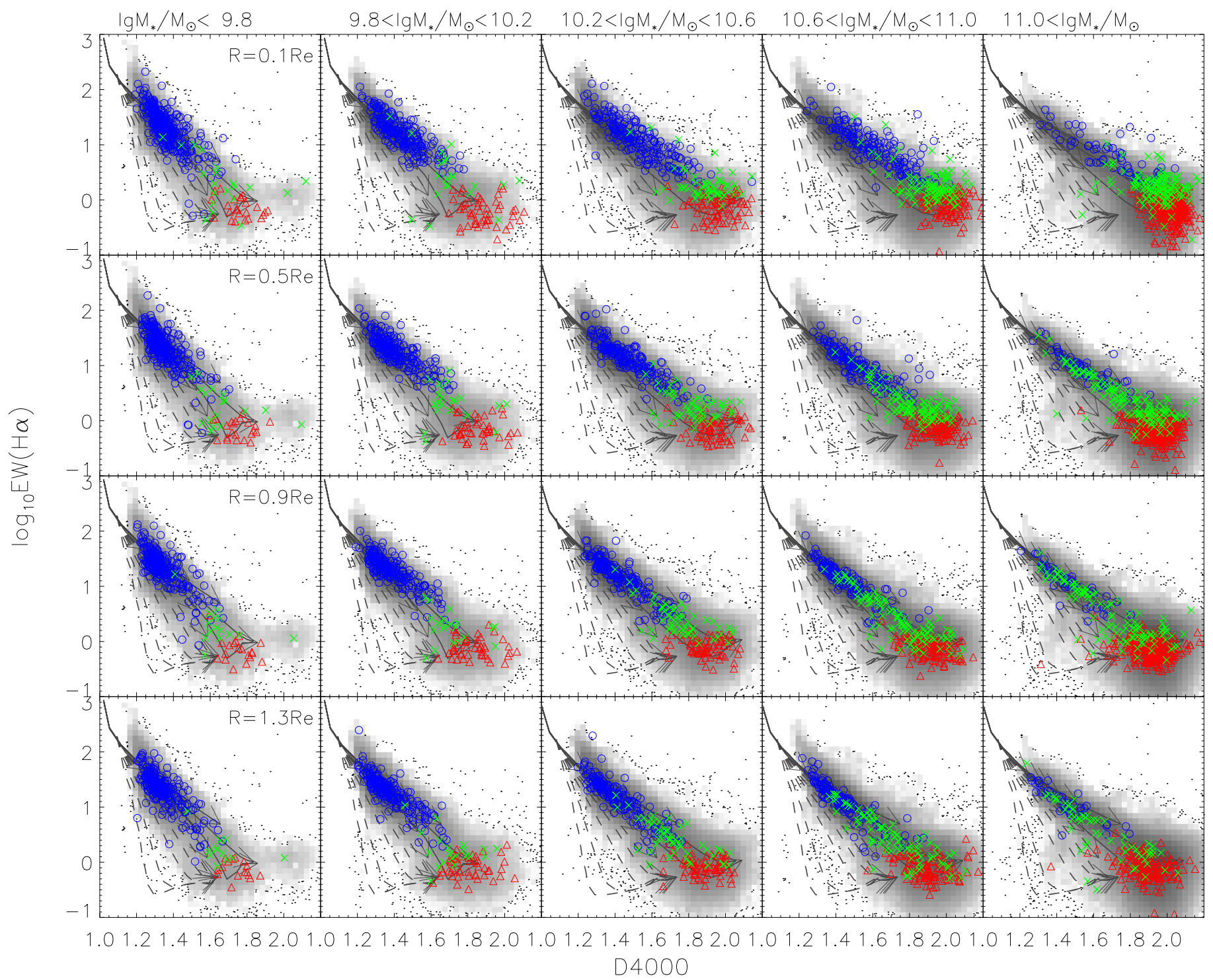

Figure 11. Same as Figure 9 but showing distributions in the plane of $\mathrm{D}_{n}(4000)$ and $\log _{10} \mathrm{EW}(\mathrm{H} \alpha)$.

\subsection{A Critical Mass for Inside-out Star Formation Cessation}

The radial gradients in the SFH diagnostics as measured from the MaNGA galaxies have revealed an inside-out picture of star formation cessation. This can be seen from both the radial profiles of the three diagnostic parameters, as shown in Figure 6, and the comparisons of the inner and outer $\mathrm{D}_{n}(4000)$, as shown in Figure 12. The diagnostic diagrams examining the mutual relations of the three parameters, as shown in Figures 9-11, further indicate that this inside-out cessation must happen in a rather smooth manner, with the SFR declining continuously on a long timescale. In this picture, as discussed in Section 3.4, a fully SF galaxy with a low stellar mass and weak/no gradients in $\mathrm{D}_{n}(4000)$ will evolve into the PQ phase with a quenched inner region and an SF outer region, thus displaying significantly negative gradients in stellar age, and eventually to the final, fully quenched stage with a larger stellar mass and only weak/no gradients again.

When we limit the analysis to a narrow range of stellar mass (see Figure 13), we find that the picture of inside-out cessation appears to hold only for galaxies with stellar masses above $\sim 10^{10} M_{\odot}$. For less massive galaxies, the inner and outer regions seem to evolve synchronously, showing almost no gradients at all in the evolutionary stages as classified by the $f_{Q}$ parameter. This finding implies that inside-out cessation is at work in a galaxy only when its stellar mass exceeds a critical mass, which is a few $\times 10^{10} M_{\odot}$. A galaxy with an initial stellar mass below this critical mass may evolve to its fate via two different paths, depending on the final mass. If the final mass is below the critical mass, star formation would cease synchronously across the galaxy, thus showing no/weak radial gradients over the galaxy lifetime. If the galaxy manages to keep forming stars over a long timescale so that its stellar mass exceeds the critical mass, or if the galaxy begins with a mass above the critical value, inside-out star formation cessation would then be at work. For a fully quenched galaxy with a current mass below the critical value, star formation must have ceased in a synchronous manner with no radial dependence.

We have further examined whether the onset of inside-out cessation depends on the $\mathrm{B} / \mathrm{T}$ luminosity ratio and the spheroid/disk classification. It is interesting that, although the relative fraction of galaxies falling in the subsets of SF, $\mathrm{PQ}$, and TQ may vary according to $f_{\mathrm{B} / \mathrm{T}}$ or morphology, the overall 

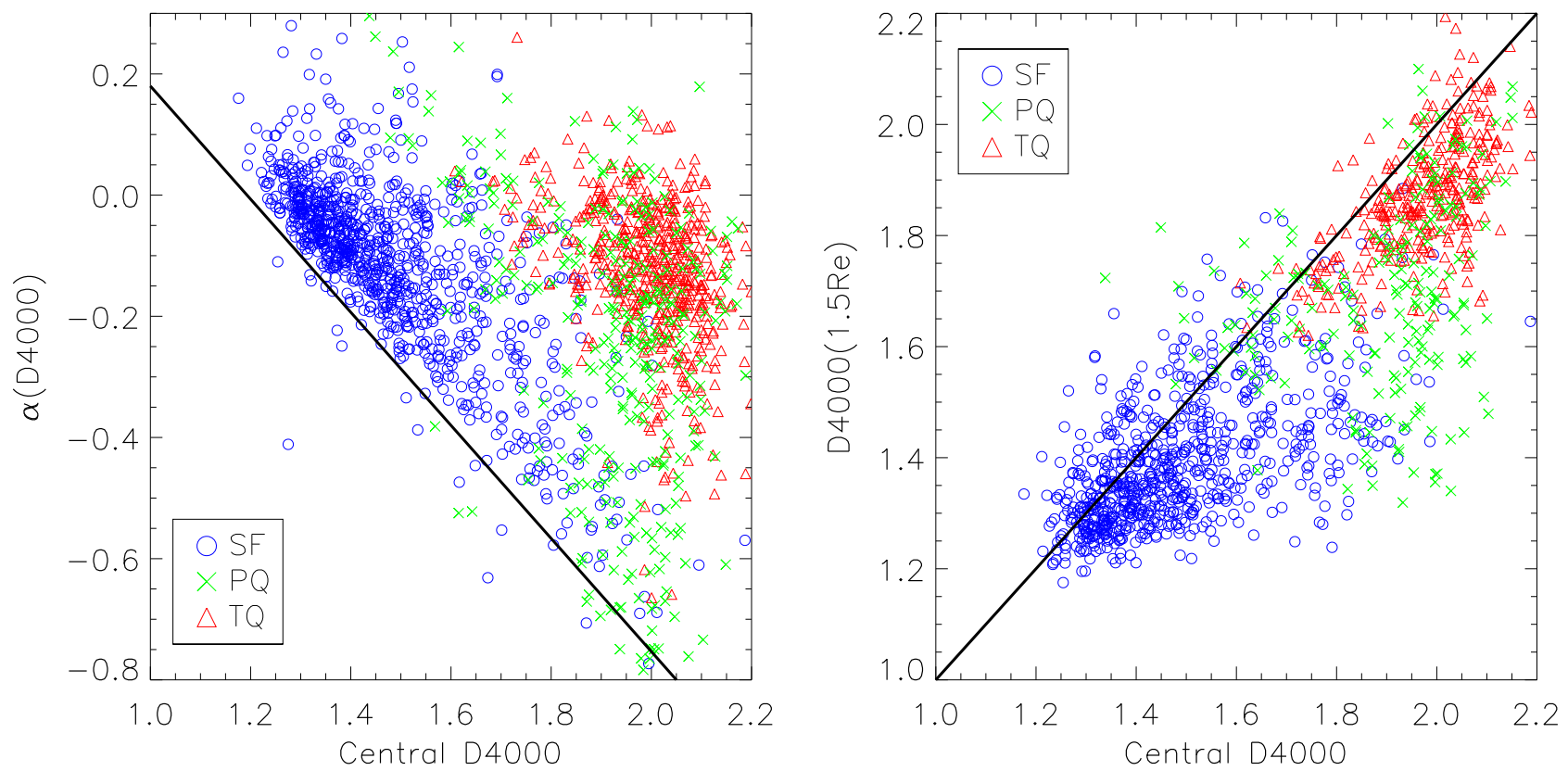

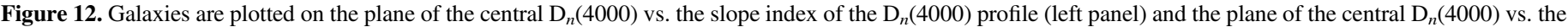

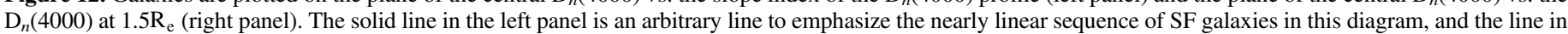

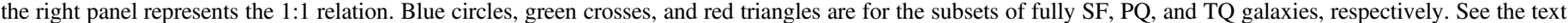
for a detailed description of the classification method.

distribution of galaxies on the $\mathrm{D}_{n}(4000)_{1.5 \mathrm{Re}}-\mathrm{D}_{n}(4000)_{\text {cen }}$ plane appears to depend on stellar mass only. At higher masses, all types of galaxies show larger radial variations in $\mathrm{D}_{n}(4000)$, regardless of their morphology type or $\mathrm{B} / \mathrm{T}$ ratio. This analysis demonstrates that stellar mass is indeed a driving parameter for the star formation cessation process in galaxies.

\subsection{Galactic Bulge: Reason for or Result of Star Formation Cessation?}

One might argue that the results regarding inside-out cessation as discussed above can be interpreted by the increasing predominance of galactic bulges with increasing stellar mass, which may naturally explain the $\mathrm{D}_{n}(4000)$ gradients observed in massive galaxies. Figure 14 shows that galaxies with a small bulge luminosity fraction $\left(f_{\mathrm{B} / \mathrm{T}}<0.4\right)$ present stronger $\mathrm{D}_{n}(4000)$ gradients at higher masses in exactly the same way as seen for the bulge-dominated galaxies with $f_{\mathrm{B} / \mathrm{T}}>0.4$. Therefore, the inside-out cessation of star formation as observed in the massive galaxies cannot be solely attributed to bulge growth. In the Appendix, we present an additional analysis in which we select galaxies with a bulge radius $R_{\text {bulge }}$ smaller than $0.5 R_{\mathrm{e}}$, thus estimating $\mathrm{D}_{n}(4000)$ gradients using only the disk-dominated regions, and we find significant mass-dependent gradients similar to those seen in the previous section.

Previous studies have established that the presence of a central dense object (such as a prominent bulge) is a necessary but insufficient condition for a central galaxy to be quenched (e.g., Bell 2008; Bell et al. 2012; Fang et al. 2013). Bluck et al. (2014) further found the bulge mass to play a more important role in quenching star formation in local galaxies when compared to the $\mathrm{B} / \mathrm{T}$ stellar mass ratio. In agreement with these previous studies, Figure 14 shows that the majority of the TQ galaxies in our sample have $f_{\mathrm{B} / \mathrm{T}}>0.4$, but, on the other hand, only a fraction of the galaxies with $f_{\mathrm{B} / \mathrm{T}}>0.4$ are fully quenched.

However, we would like to point out that this agreement does not necessarily mean that a massive bulge is a necessary condition or a driver for star formation quenching. Rather, these results show that the bulge is just a natural by-product of the evolution process. This can be clearly seen from Figure 14, where deviations of massive galaxies from the 1:1 relation, indicative of inside-out cessation, can happen in all the galaxies with a central $\mathrm{D}_{n}(4000)$ larger than $\sim 1.4$, no matter whether the galaxy has a prominent bulge or not. In fact, the deviation from the 1:1 relation happens quite early, when the galaxy is at the SF stage without a prominent bulge, and a large $\mathrm{B} / \mathrm{T}$ ratio is observed mostly when it is approaching the final stage to become a fully quenched galaxy. If a prominent bulge is a necessary condition for driving the cessation process, the deviation should be observed only in the upper panels of the figure, where the galaxies have a large $f_{\mathrm{B} / \mathrm{T}}$. For less massive galaxies, which may not follow the inside-out cessation process, a large $f_{\mathrm{B} / \mathrm{T}}$ is unlikely to be a necessary condition either, as we see TQ galaxies in the lower panels with $f_{\mathrm{B} / \mathrm{T}}<0.4$ that are not a negligible population, considering the small number of PQ galaxies in both the upper and lower panels at low masses.

We conclude that the presence of a central dense object, such as a bulge, is a by-product but not a driving factor of star formation cessation in local galaxies. It is possible that, when fully grown, the bulge can play some role in finalizing the cessation process, perhaps by suppressing bar formation or even destroying the bar structure in the galactic center, which is believed to be the primary driver of the secular evolution. Numerical studies predicted that the central random motions can stabilize the disk against bar formation (Athanassoula \& Sellwood 1986; Athanassoula 2008), and an anticorrelation of bar fraction with central stellar velocity dispersion has been 

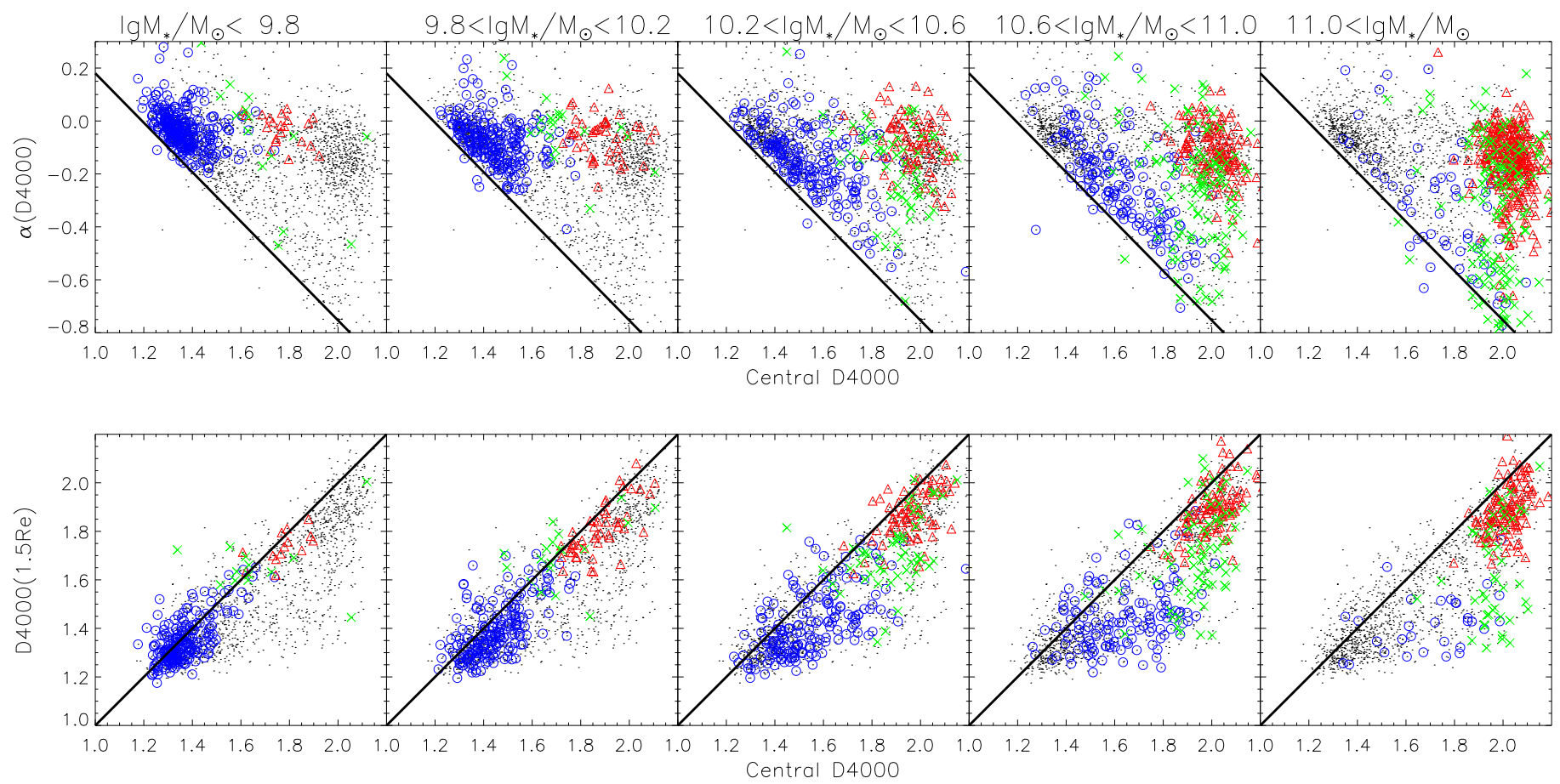

Figure 13. Top (bottom) panels are the same as the left (right) panels in the previous figure, with the different panels for five stellar mass intervals.

observed for both low- $z$ and high- $z$ galaxies (Das et al. 2008; Sheth et al. 2012; Cervantes-Sodi et al. 2013).

\section{Conclusions}

We have investigated the resolved star formation histories of 1917 local galaxies with regular morphology using integral field spectroscopy from the MaNGA MPL5. We have obtained both two-dimensional maps and radial profiles of three parameters, $\mathrm{D}_{n}(4000), \mathrm{EW}\left(\mathrm{H} \delta_{A}\right)$ and $\mathrm{EW}(\mathrm{H} \alpha)$, which combine to provide powerful diagnostics of the recent SFH. Based on the $\mathrm{D}_{n}(4000)$ and $\mathrm{EW}\left(\mathrm{H} \delta_{A}\right)$ maps, we propose a new parameter, $f_{Q}\left(1.5 \mathrm{R}_{\mathrm{e}}\right)$, to quantify the overall star formation status of a galaxy, defined as the stellar mass-weighted fraction of the quenched spaxels within $1.5 \mathrm{R}_{\mathrm{e}}$. Accordingly, we divide our galaxies into three subsets: fully $\mathrm{SF}\left(f_{Q}\left(1.5 \mathrm{R}_{\mathrm{e}}\right)<0.1\right)$, PQ $\left(0.1 \leqslant f_{Q}\left(1.5 \mathrm{R}_{\mathrm{e}}\right)<0.9\right)$, and TQ $\left(f_{Q}\left(1.5 \mathrm{R}_{\mathrm{e}}\right) \geqslant 0.9\right)$. We then study the radial gradients in the SFH diagnostic parameters, comparing the results for the three types and for galaxies with different stellar masses and structural parameters.

Our conclusions can be summarized as follows.

1. Less massive galaxies with stellar mass below $\sim 10^{10} M_{\odot}$ present no or very weak gradients in all of the diagnostic parameters, regardless of the overall star formation status as quantified by $f_{Q}\left(1.5 \mathrm{R}_{\mathrm{e}}\right)$, but the amplitudes of the profiles vary with $f_{Q}\left(1.5 \mathrm{R}_{\mathrm{e}}\right)$, with older stellar populations being more dominant at larger $f_{Q}$.

2. Massive galaxies with stellar mass above $\sim 10^{10} M_{\odot}$ present significant gradients in all three diagnostics if classified as an SF or PQ galaxy but show weak gradients in both $\mathrm{D}_{n}(4000)$ and $\mathrm{EW}\left(\mathrm{H} \delta_{A}\right)$ and no gradients in $\mathrm{EW}(\mathrm{H} \alpha)$ if classified as a TQ galaxy. The observed gradients are stronger with increasing mass.

3. The majority of the spaxels of the sample galaxies closely follow the continuous star formation models of $\mathrm{BC} 03$, regardless of stellar mass and $f_{Q}\left(1.5 \mathrm{R}_{\mathrm{e}}\right)$, indicating that ongoing starbursts are very rare in galaxies with regular morphology.

4. Distributions of the SF, PQ, and TQ populations on the plane of the central $\mathrm{D}_{n}(4000)$ versus $\mathrm{D}_{n}(4000)$ gradient or the central $\mathrm{D}_{n}(4000)$ versus $\mathrm{D}_{n}(4000)$ at $1.5 \mathrm{R}_{\mathrm{e}}$ reveal a critical stellar mass, $\sim 10^{10} M_{\odot}$, above which star formation cessation in a galaxy happens from the inside out. Galaxies tend to evolve synchronously at all radii before their mass reaches the critical mass.

5. The above conclusion holds for galaxies with or without a significant bulge and with spheroid-like or disklike morphologies. This indicates that the presence of a central dense object is not a driving parameter but rather a by-product of the star formation cessation process.

The large sample of galaxies with integral field spectroscopy from the ongoing MaNGA survey has enabled us to extensively analyze the radial gradients in the recent SFH of local galaxies and examine the dependence on both stellar mass and structural properties. This has led to the finding of a critical mass, which divides galaxies into two distinct classes following different star formation cessation processes. We would like to emphasize that more work is needed in order to better understand the implications and limitations of our results, which are limited to galaxies with regular morphologies. That will be the purpose of the next papers in this series.

This work is supported by the National Key Basic Research Program of China (No. 2015CB857004). EW is supported by the Youth Innovation Fund by the University of Science and Technology of China (No. WK2030220019), the China Postdoctoral Science Foundation (No. BH2030000040), and NSFC (grant No. 11421303). CL acknowledges the support of NSFC (grant Nos. 11173045, 11233005, 11325314, and 11320101002) and the Strategic Priority Research Program "The Emergence of Cosmological Structures" of CAS (grant No. XDB09000000). MAB acknowledges support from NSF 


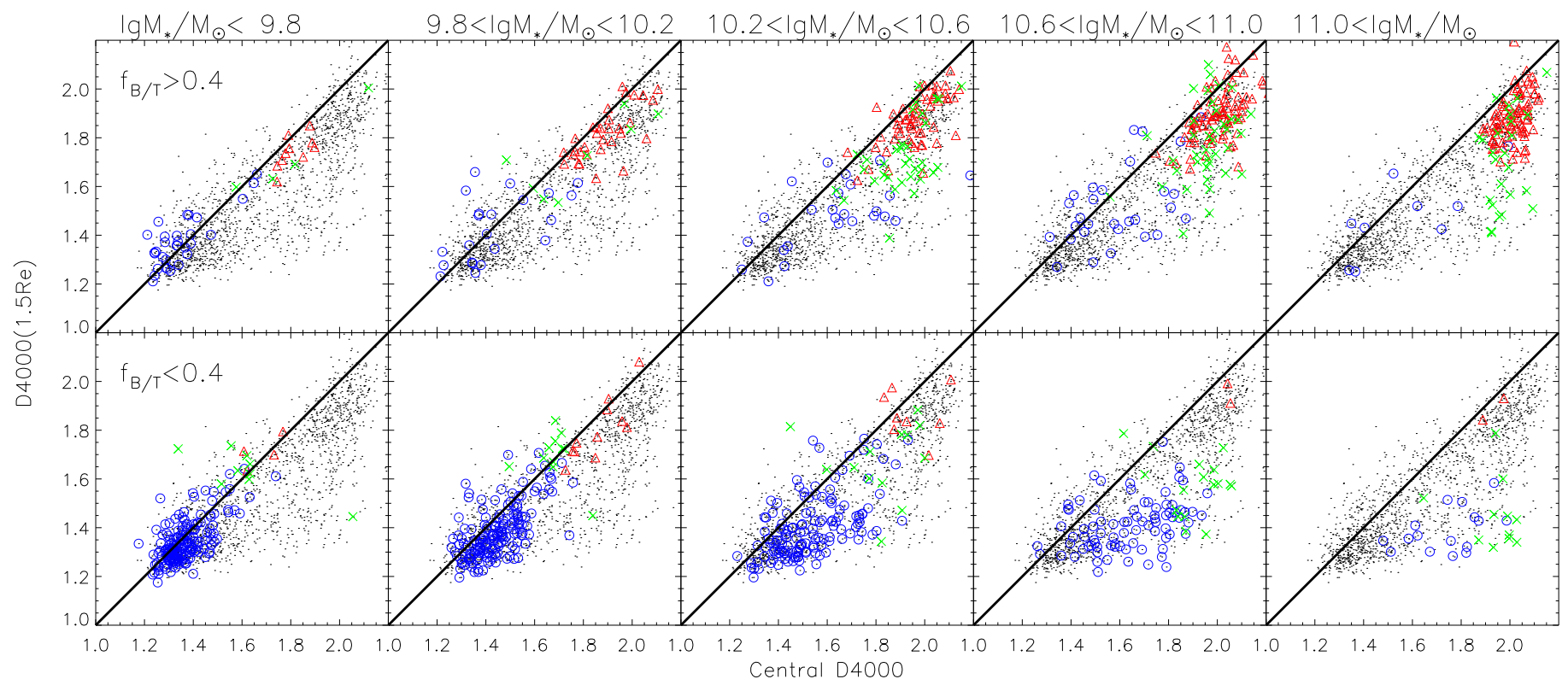

Figure 14. Correlation between the central $4000 \AA$ break and the $4000 \AA$ break at $1.5 \mathrm{R}_{\mathrm{e}}$. Panels from left to right correspond to different stellar mass ranges, as indicated above the top panels. The top panels show results for galaxies with $f_{\mathrm{B} / \mathrm{T}}>0.4$, and the bottom panels show results for those with $f_{\mathrm{B} / \mathrm{T}}<0.4$. Colors and symbols are the same as in Figure 12.

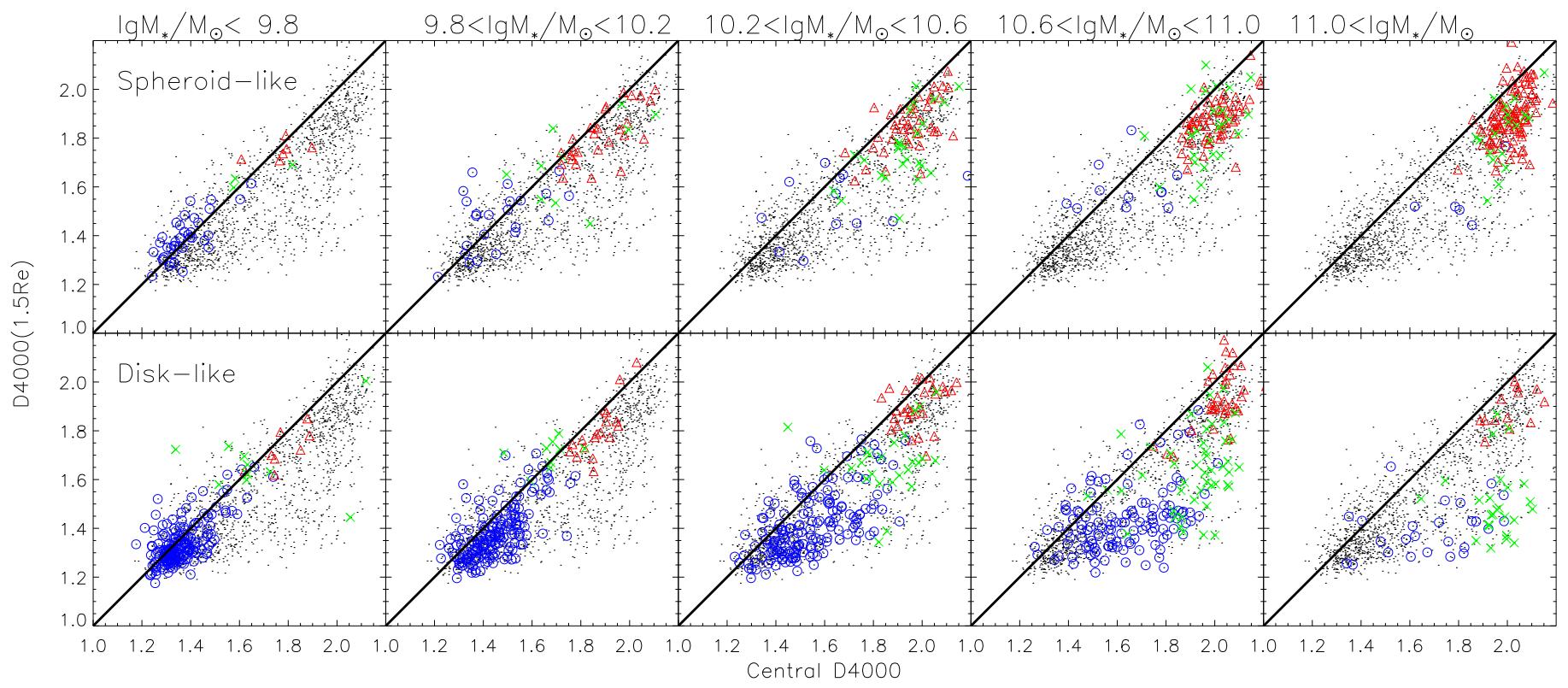

Figure 15. Correlation between the central $4000 \AA$ break and the $4000 \AA$ break at $1.5 \mathrm{R}_{\mathrm{e}}$. Panels from left to right correspond to different stellar mass ranges, as indicated above the top panels. The top panels show results for galaxies with spheroid-like morphology, and the bottom panels show results for those with disklike morphology. Colors and symbols are the same as Figure 12.

AST-1517006. RR thanks CNPq and FAPERGS for partial financial support.

Funding for SDSS-IV has been provided by the Alfred P. Sloan Foundation, the US Department of Energy Office of Science, and the Participating Institutions. SDSS-IV acknowledges support and resources from the Center for HighPerformance Computing at the University of Utah. The SDSS website is http://www.sdss.org.

SDSS-IV is managed by the Astrophysical Research Consortium for the Participating Institutions of the SDSS Collaboration, including the Brazilian Participation Group, the Carnegie Institution for Science, Carnegie Mellon University, the Chilean Participation Group, the French Participation Group, the Harvard-Smithsonian Center for Astrophysics, Instituto de Astrofísica de Canarias, the Johns Hopkins University, Kavli Institute for the Physics and Mathematics of the Universe (IPMU)/University of Tokyo, Lawrence Berkeley National Laboratory, Leibniz Institut für Astrophysik Potsdam (AIP), Max-Planck-Institut für Astronomie (MPIA Heidelberg), Max-Planck-Institut für Astrophysik (MPA Garching), Max-Planck-Institut für Extraterrestrische Physik (MPE), the National Astronomical Observatory of China, New Mexico State University, New York University, the University of Notre Dame, Observatório Nacional/MCTI, the Ohio State 

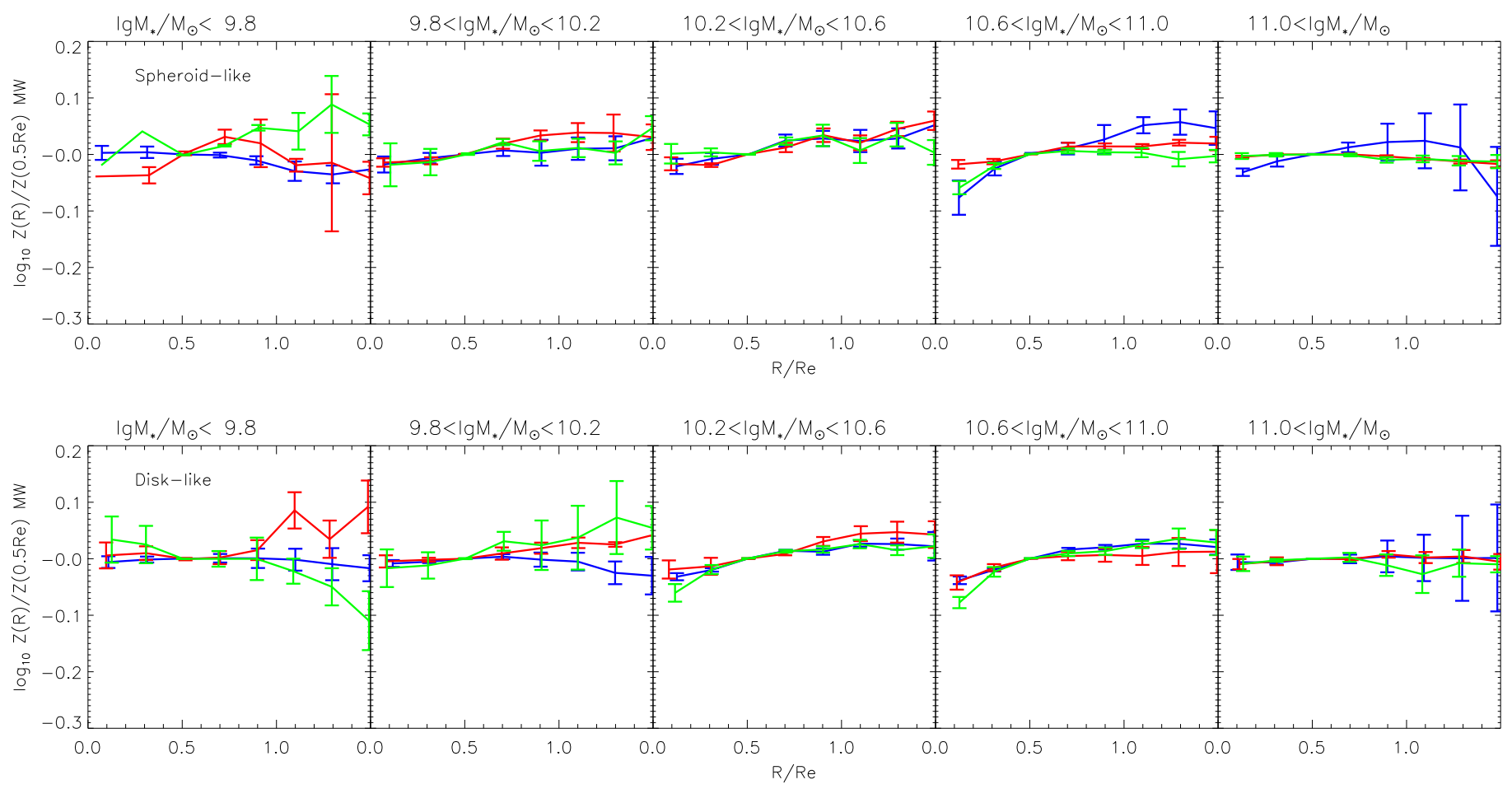

Figure 16. Median radial gradients of mass-weighted stellar metallicity for the SF (blue curves), PQ (green curves), and TQ (red curves) populations. The gradients are displayed with increasing stellar mass bins from left to right for spheroid-like (top panels) and disklike (bottom panels) galaxies. The colors and symbols are the same as in Figure 8. The errors are measured by the bootstrap method.

University, Pennsylvania State University, the Shanghai Astronomical Observatory, the United Kingdom Participation Group, Universidad Nacional Autónoma de México, the University of Arizona, the University of Colorado Boulder, the University of Oxford, the University of Portsmouth, the University of Utah, the University of Virginia, the University of Washington, the University of Wisconsin, Vanderbilt University, and Yale University.

\section{Appendix}

\section{A.1. The Metallicity and Stellar Age Profiles from STARLIGHT}

In Figure 16, we present the gradients of mass-weighted stellar metallicity for SF (blue curves), PQ (green curves), and TQ (red curves) galaxies in the five stellar mass bins. The gradients of metallicity are presented for spheroid-like and disklike galaxies separately. The stellar metallicities are from the STARLIGHT output. As shown, no or weak radial variations in stellar metallicity are found in almost all of the subsamples. Both spheroid-like and disklike galaxies with $10.2<\log _{10}\left(M_{*} / M_{\odot}\right)<11.0$ are found to have slightly positive gradients regardless of their quenching statuses, while galaxies with $\log _{10}\left(M_{*} / M_{\odot}\right)<9.8$ and $\log _{10}\left(M_{*} / M_{\odot}\right)>11.0$ appear to have flat radial profiles of stellar metallicity as a whole. We note that it is difficult to measure the stellar metallicity in an accurate way, since the measurements of stellar metallicity largely depend on the spectral fitting procedures.

Since all of our results are based on the three diagnostic parameters, here we try to examine our results by using the mean stellar age. Figure 17 shows the median radial gradients of light-weighted stellar ages for SF (blue curves), PQ (green curves), and TQ (red curves) galaxies for the five stellar mass bins. Similarly, we present these for spheroid-like and disklike galaxies separately. The light-weighted stellar ages are derived from STARLIGHT measurements. As can be seen from Figure 17, the stellar age of disklike TQ galaxies exhibits weak radial variations for both low and high stellar mass bins. In contrast, the disklike SF and PQ galaxies show pronounced age gradients for high stellar mass bins and no or weak radial variations for low stellar mass bins. In addition, these age gradients increase with increasing stellar mass monotonously. However, the spheroid-like galaxies exhibit weak radial variations of stellar age, and their stellar age gradients do not show significant differences for the SF, PQ, and TQ populations. In general, all of these results are well consistent with what we have found in Figures 6 and 8.

In addition, we compare the mean stellar age at the galactic centers with that at the $1.5 \mathrm{R}_{\mathrm{e}}$ radial bins in Figure 18 . We present the light-weighted age comparisons in the top row and the mass-weighted age comparisons in the bottom row for SF, $\mathrm{PQ}$, and TQ galaxies in the same five stellar mass bins. As expected, the mean stellar age increases from SF to PQ to TQ populations in both high and low stellar mass bins. Thus, we can illustrate these panels by assuming that galaxies evolve from SF to PQ to TQ status. As seen from the top row, the stellar populations in the inner and outer regions become old simultaneously for less massive galaxies. However, for massive galaxies, the stellar population in the central regions get old first, then it extends to larger and larger radii. This confirms our previous result that star formation cessation occurs in the inner and outer regions simultaneously for less massive galaxies, while it occurs first in galactic centers and then extends to larger and larger radii for massive galaxies.

In the bottom panels of Figure 18, there is a good linear relation between the mass-weighted age at galactic center and the $1.5 \mathrm{R}_{\mathrm{e}}$ radial bins in the logarithmic space in each stellar 

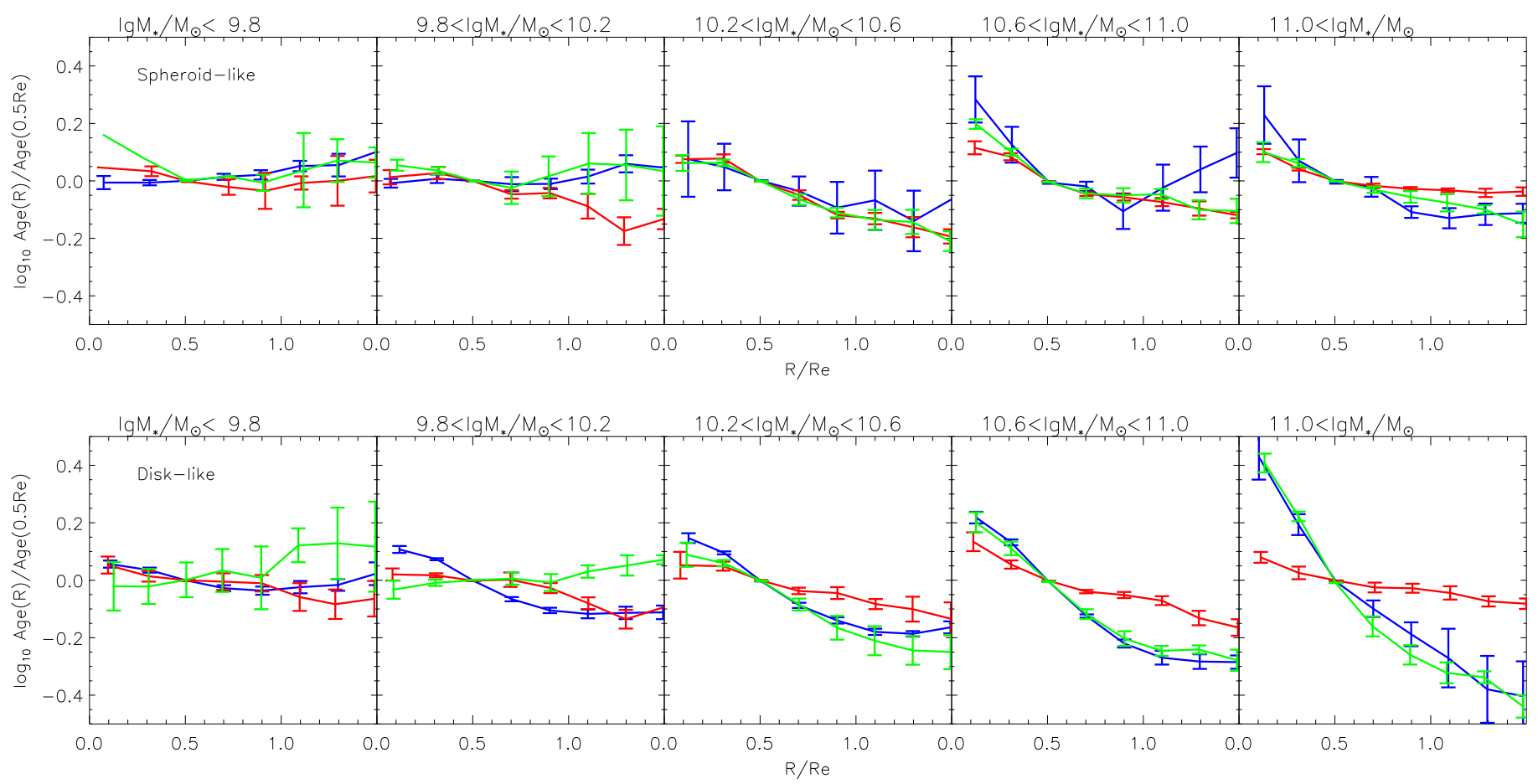

Figure 17. Median radial gradients of light-weighted stellar ages for the SF (blue curves), PQ (green curves), and TQ (red curves) populations. The gradients are displayed with increasing stellar mass bins from left to right for spheroid-like (top panels) and disklike (bottom panels) galaxies. The colors and symbols are the same as in Figure 8. The errors are measured by the bootstrap method.
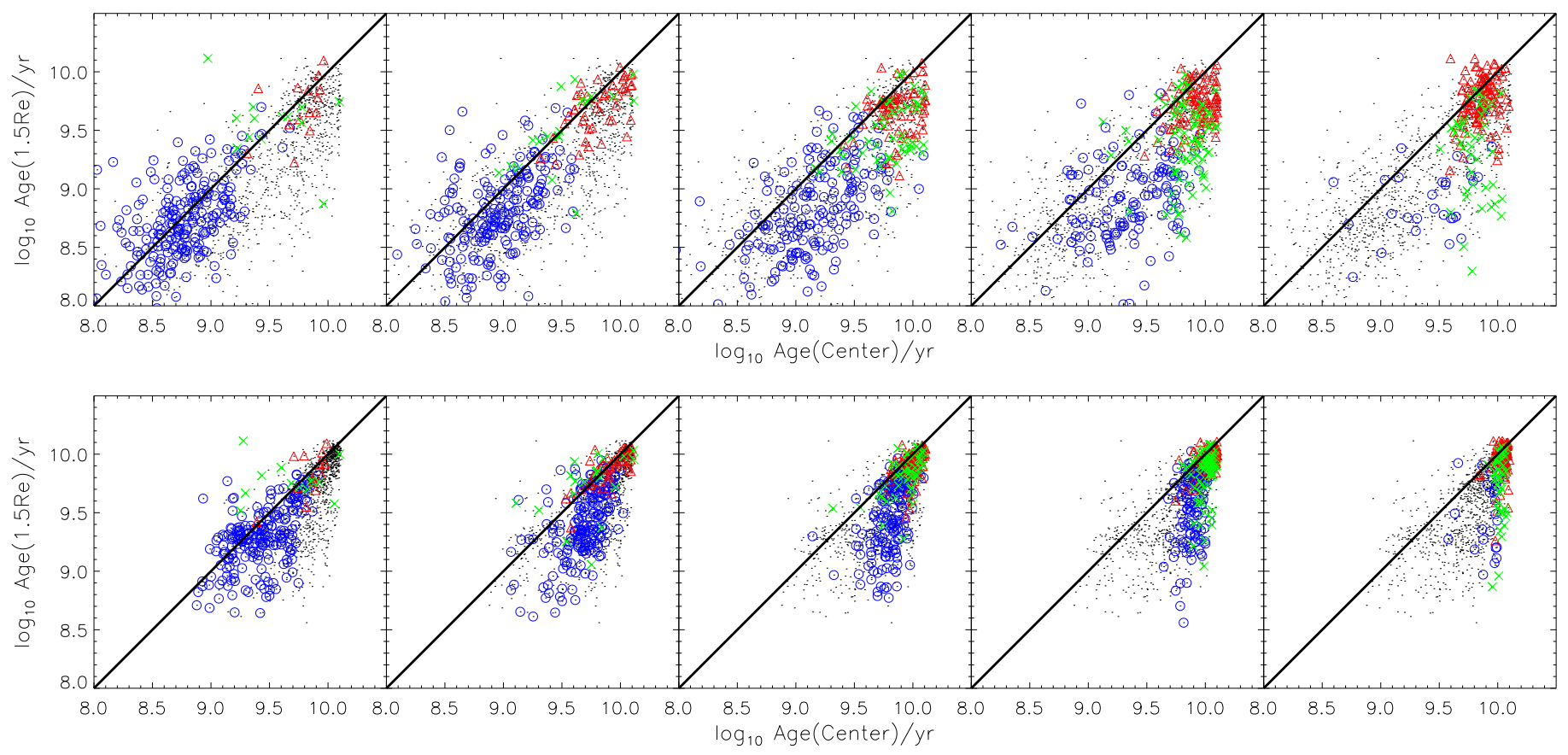

Figure 18. Comparison between mean stellar age at the galactic center and $1.5 \mathrm{R}_{\mathrm{e}}$ radial bin in five stellar mass bins. We present these comparisons by using lightweighted (top panels) and mass-weighted (bottom panels) stellar age, respectively. Colors and symbols are the same as in Figure 13. The black line in each panel is the one-to-one line. For comparison, all galaxies in our sample are plotted as small gray dots in each panel.

mass bin. With increasing stellar mass, this relation becomes steeper and steeper and the scatter gets to be smaller and smaller in the logarithmic space. For SF galaxies, massweighted stellar ages are significantly larger in the galactic center than in the $1.5 \mathrm{R}_{\mathrm{e}}$ radial bins, especially for massive galaxies ( $\sim 10.0 \mathrm{Gyr})$. However, this is not the case for lightweighted stellar ages. Although some SF galaxies are forming new stars in their centers, there has already been a dominant fraction of old stars formed in the past several Gyr. Compared to the inner regions, the outer regions are dominated by young stars, which reveals an inside-out growth scenario.

We note that the flat metallicity profiles appear to be inconsistent with the previous results in the literature. However, we have verified that this issue is not caused by the MaNGA data 


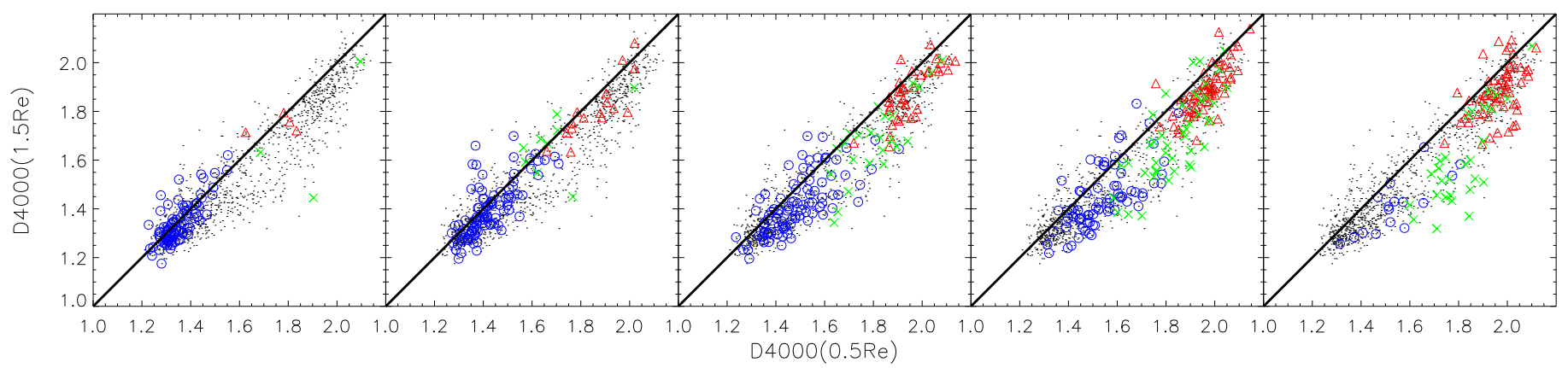

Figure 19. The $\mathrm{D}_{n}(4000)_{0.5 \mathrm{Re}}$ vs. $\mathrm{D}_{n}(4000)_{1.5 \mathrm{Re}}$ plane for galaxies with $0.5 \mathrm{R}_{\mathrm{e}}$ greater than $R_{\text {bulge. }}$. We present this figure by separating the galaxies into the five stellar mass bins, as above. The symbols, colors, and lines are the same as in Figure 14.
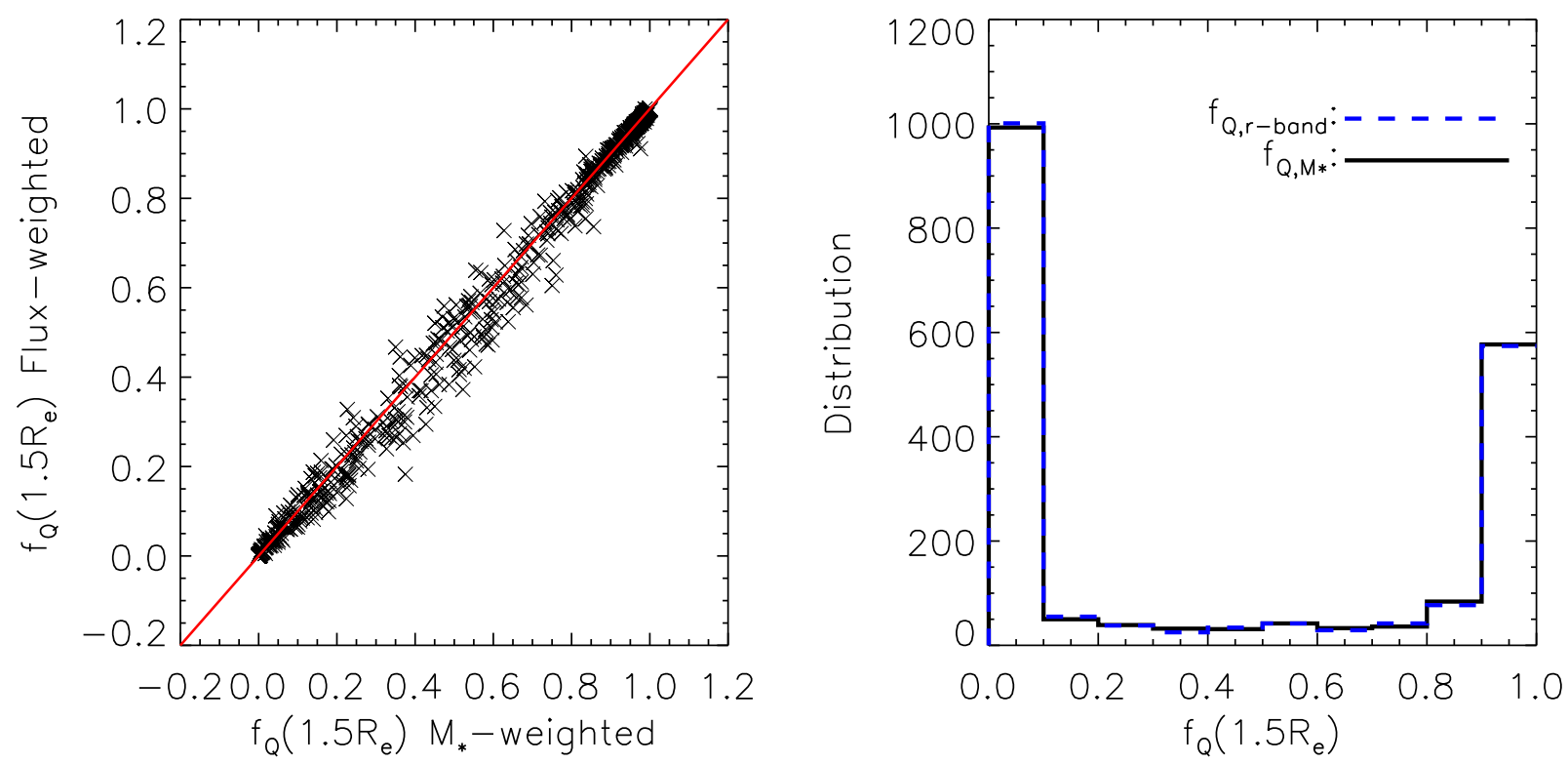

Figure 20. Left panel: comparison of $f_{Q, M_{*}}$ vs. $f_{Q, \text { flux }}$. The red line is the one-to-one relation. Right panel: distribution of $f_{Q, M_{*}}(1.5 \mathrm{Re})$ and $f_{Q, \text { flux }}(1.5 \mathrm{Re})$ for the sample galaxies.

themselves. For this, we have reproduced Figures 16-18 using the stellar age and metallicity maps produced by the Pipe3D pipeline (Sánchez et al. 2016a, 2016b), which are publicly available as a value-added catalog from the SDSS DR14 at http://www.sdss.org/dr14/manga/manga-data/manga-pipe3dvalue-added-catalog/. We find negative metallicity gradients for massive galaxies, consistent with previous results. More importantly, the stellar age gradients are in good agreement with those from STARLIGHT, indicating that the $\mathrm{D}_{n}(4000)$ gradients presented in the main text are not dominated by possible gradients in metallicity.

\section{A.2. The $D_{n}$ (4000) Gradients for Disk-dominated Regions}

As proposed in Section 4.3, quenched galaxies are usually found to have massive bulges. One question arises: are the $\mathrm{D}_{n}(4000)$ or stellar age gradients in massive SF and PQ galaxies mainly due to the combination effect of a old massive bulge and a young stellar disk? To answer this question, we first select galaxies with $0.5 \mathrm{R}_{\mathrm{e}}$ greater than the bulge size $\left(R_{\text {bulge }}\right)$. The bulge size is taken from Simard et al. (2011). Then, we compare the $\mathrm{D}_{n}(4000)$ in the $0.5 \mathrm{R}_{\mathrm{e}}$ and $1.5 \mathrm{R}_{\mathrm{e}}$ radial bins to figure out whether the gradient in $\mathrm{D}_{n}(4000)$ is retained or not between $0.5 \mathrm{R}_{\mathrm{e}}$ and $1.5 \mathrm{R}_{\mathrm{e}}$ (the bin width is $0.2 \mathrm{R}_{\mathrm{e}}$ ). For the galaxies we selected, the regions between $0.5 \mathrm{R}_{\mathrm{e}}$ and $1.5 \mathrm{R}_{\mathrm{e}}$ are dominated by a disk component. Figure 19 shows the $\mathrm{D}_{n}(4000)_{0.5 \mathrm{Re}}$ versus $\mathrm{D}_{n}(4000)_{1.5 \mathrm{Re}}$ plane for galaxies with $0.5 R_{\mathrm{e}}$ greater than $R_{\text {bulge }}$. We find that pronounced gradients still exist for massive SF and PQ galaxies even for disk-dominated regions, which suggests that the existence of a massive bulge is not the only reason for the observed $\mathrm{D}_{n}(4000)$ gradients, and disk components exhibit significant gradients in $\mathrm{D}_{n}(4000)$ (or in light-weighted stellar age).

\section{A.3. The Definition of the Quenched Fraction Weighting by r-band Flux}

In the main text, we introduced a parameter to describe the quenching status for each galaxy, the quenched fraction $\left(f_{Q, M_{*}}\right)$, which is defined as the mass-weighted fraction of spaxels that are quenched within a given galactic radius. Since the stellar masses of individual spaxels are model-dependent to some degree, we try to weight the spaxels with $r$-band flux in defining the quenched fraction $\left(f_{Q \text {,flux }}\right)$. The left panel of Figure 20 shows the comparison of $f_{Q, M_{*}}(1.5 \mathrm{Re})$ and $f_{Q \text {,flux }}(1.5 \mathrm{Re})$ for the sample galaxies. As shown, the flux-weighted quenched fraction is in good linear correlation with the mass-weighted quenched fraction. We note that there are many data points in the low- $f_{Q}$ end and high- $f_{Q}$ end, since the majority of galaxies are either SF or TQ. The right panel of Figure 20 shows the distribution of 
$f_{Q, M_{*}}(1.5 \mathrm{Re})$ and $f_{Q \text {,flux }}(1.5 \mathrm{Re})$ for the sample galaxies. As shown, the distributions of the two are almost the same. This indicates that using the flux-weighted quenching definition would not change our results. In addition, we present a modelindependent definition of the quenched fraction weighting by $r$-band flux, which will be adopted in our following work.

\section{ORCID iDs}

Enci Wang (ㄱ) https://orcid.org/0000-0003-1588-9394 Lin Lin (1) https://orcid.org/0000-0003-1138-8146 Matthew Bershady (i) https://orcid.org/0000-0002-3131-4374 David R. Law (1) https://orcid.org/0000-0002-9402-186X Michael Merrifield (1) https://orcid.org/0000-0002-4202-4727 Sebastian F. Sanchez (1) https://orcid.org/0000-00016444-9307

Renbin Yan (1) https://orcid.org/0000-0003-1025-1711

\section{References}

Abadi, M. G., Moore, B., \& Bower, R. G. 1999, MNRAS, 308, 947 Abolfathi, B., Aguado, D. S., Aguilar, G., et al. 2017, ApJS, in press (arXiv:1707.09322)

Abraham, R. G., Ellis, R. S., Fabian, A. C., Tanvir, N. R., \& Glazebrook, K. 1999, MNRAS, 303, 641

Athanassoula, E. 2008, MNRAS, 390, L69

Athanassoula, E., \& Sellwood, J. A. 1986, MNRAS, 221, 213

Bacon, R., Copin, Y., Monnet, G., et al. 2001, MNRAS, 326, 23

Baldwin, J. A., Phillips, M. M., \& Terlevich, R. 1981, PASP, 93, 5

Balogh, M. L., Navarro, J. F., \& Morris, S. L. 2000, ApJ, 540, 113

Barro, G., Faber, S. M., Koo, D. C., et al. 2017, ApJ, 840, 47

Belfiore, F., Maiolino, R., Bundy, K., et al. 2015, MNRAS, 449, 867

Belfiore, F., Maiolino, R., Maraston, C., et al. 2016, MNRAS, 461, 3111

Belfiore, F., Maiolino, R., Maraston, C., et al. 2017, MNRAS, 466, 2570

Bell, E. F. 2008, ApJ, 682, 355

Bell, E. F., \& de Jong, R. S. 2000, MNRAS, 312, 497

Bell, E. F., McIntosh, D. H., Barden, M., et al. 2004, ApJL, 600, L11

Bell, E. F., van der Wel, A., Papovich, C., et al. 2012, ApJ, 753, 167

Bershady, M. A., Verheijen, M. A. W., Swaters, R. A., et al. 2010, ApJ, 716, 198

Birnboim, Y., \& Dekel, A. 2003, MNRAS, 345, 349

Blanc, G. A., Weinzirl, T., Song, M., et al. 2013, AJ, 145, 138

Blanton, M. R., Bershady, M. A., Abolfathi, B., et al. 2017, AJ, 154, 28

Blanton, M. R., Kazin, E., Muna, D., Weaver, B. A., \& Price-Whelan, A. 2011, AJ, 142, 31

Bluck, A. F. L., Mendel, J. T., Ellison, S. L., et al. 2014, MNRAS, 441, 599

Blumenthal, G. R., Faber, S. M., Primack, J. R., \& Rees, M. J. 1984, Natur, 311,517

Brinchmann, J., Charlot, S., White, S. D. M., et al. 2004, MNRAS, 351, 1151

Brodie, J. P., Romanowsky, A. J., Strader, J., et al. 2014, ApJ, 796, 52

Bruzual, G., \& Charlot, S. 2003, MNRAS, 344, 1000

Bundy, K., Bershady, M. A., Law, D. R., et al. 2015, ApJ, 798, 7

Bundy, K., Ellis, R. S., Conselice, C. J., et al. 2006, ApJ, 651, 120

Bundy, K., Scarlata, C., Carollo, C. M., et al. 2010, ApJ, 719, 1969

Cappellari, M., Emsellem, E., Krajnović, D., et al. 2011, MNRAS, 416, 1680

Catalán-Torrecilla, C., Gil de Paz, A., Castillo-Morales, A., et al. 2017, ApJ, 848,87

Cattaneo, A., Dekel, A., Devriendt, J., Guiderdoni, B., \& Blaizot, J. 2006, MNRAS, 370, 1651

Cervantes-Sodi, B., Li, C., Park, C., \& Wang, L. 2013, ApJ, 775, 19

Cheung, E., Faber, S. M., Koo, D. C., et al. 2012, ApJ, 760, 131

Chung, A., van Gorkom, J. H., Kenney, J. D. P., Crowl, H., \& Vollmer, B. 2009, AJ, 138, 1741

Cid Fernandes, R., Mateus, A., Sodré, L., Stasińska, G., \& Gomes, J. M. 2005, MNRAS, 358, 363

Croom, S. M., Lawrence, J. S., Bland-Hawthorn, J., et al. 2012, MNRAS, 421,872

Croton, D. J., Springel, V., White, S. D. M., et al. 2006, MNRAS, 365, 11

Das, M., Laurikainen, E., Salo, H., \& Buta, R. 2008, Ap\&SS, 317, 163

de Jong, R. S. 1996, A\&A, 313, 377

de Zeeuw, P. T., Bureau, M., Emsellem, E., et al. 2002, MNRAS, 329, 513

Dekel, A., \& Birnboim, Y. 2006, MNRAS, 368, 2
Drory, N., MacDonald, N., Bershady, M. A., et al. 2015, AJ, 149, 77 Faber, S. M., Willmer, C. N. A., Wolf, C., et al. 2007, ApJ, 665, 265 Fang, J. J., Faber, S. M., Koo, D. C., \& Dekel, A. 2013, ApJ, 776, 63 Gallazzi, A., Charlot, S., Brinchmann, J., White, S. D. M., \& Tremonti, C. A. 2005, MNRAS, 362, 41

Garnett, D. R., \& Shields, G. A. 1987, ApJ, 317, 82

Geha, M., Blanton, M. R., Yan, R., \& Tinker, J. L. 2012, ApJ, 757, 85

Goddard, D., Thomas, D., Maraston, C., et al. 2017, MNRAS, 466, 4731

González Delgado, R. M., Cid Fernandes, R., Pérez, E., et al. 2016, A\&A, 590, A44

González Delgado, R. M., García-Benito, R., Pérez, E., et al. 2015, A\&A, 581, A103

González Delgado, R. M., Pérez, E., Cid Fernandes, R., et al. 2014, A\&A, 562, A47

Gonzalez-Perez, V., Castander, F. J., \& Kauffmann, G. 2011, MNRAS, 411, 1151

Gunn, J. E., \& Gott, J. R., III 1972, ApJ, 176, 1

Gunn, J. E., Siegmund, W. A., Mannery, E. J., et al. 2006, AJ, 131, 2332

Guo, R., Hao, C.-N., Xia, X. Y., Mao, S., \& Shi, Y. 2016, ApJ, 826, 30

Huang, J.-S., Faber, S. M., Willmer, C. N. A., et al. 2013, ApJ, 766, 21

Hummer, D. G., \& Storey, P. J. 1987, MNRAS, 224, 801

Hunter, D. A., \& Elmegreen, B. G. 2004, AJ, 128, 2170

Ibarra-Medel, H. J., Sánchez, S. F., Avila-Reese, V., et al. 2016, MNRAS, 463,2799

Kauffmann, G. 2015, MNRAS, 450, 618

Kauffmann, G., Heckman, T. M., Budavári, T., et al. 2007, ApJS, 173, 357

Kauffmann, G., Heckman, T. M., White, S. D. M., et al. 2003, MNRAS, 341,54

Kereš, D., Katz, N., Weinberg, D. H., \& Davé, R. 2005, MNRAS, 363, 2

Kewley, L. J., Groves, B., Kauffmann, G., \& Heckman, T. 2006, MNRAS, 372,961

Law, D. R., Cherinka, B., Yan, R., et al. 2016, AJ, 152, 83

Law, D. R., Yan, R., Bershady, M. A., et al. 2015, AJ, 150, 19

Lequeux, J., Peimbert, M., Rayo, J. F., Serrano, A., \& Torres-Peimbert, S. 1979, A\&A, 80, 155

Li, C., Kauffmann, G., Fu, J., et al. 2012, MNRAS, 424, 1471

Li, C., Wang, E., Lin, L., et al. 2015, ApJ, 804, 125

Li, C., Wang, T.-G., Zhou, H.-Y., Dong, X.-B., \& Cheng, F.-Z. 2005, AJ, 129,669

Lin, L., Jian, H.-Y., Foucaud, S., et al. 2014, ApJ, 782, 33

Ma, C.-P., Greene, J. E., McConnell, N., et al. 2014, ApJ, 795, 158

Martig, M., Bournaud, F., Teyssier, R., \& Dekel, A. 2009, ApJ, 707, 250

Masters, K. L., Mosleh, M., Romer, A. K., et al. 2010, MNRAS, 405, 783

Merluzzi, P., Busarello, G., Dopita, M. A., et al. 2013, MNRAS, 429, 1747

Moore, B., Katz, N., Lake, G., Dressler, A., \& Oemler, A. 1996, Natur, 379,613

Moran, S. M., Heckman, T. M., Kauffmann, G., et al. 2012, ApJ, 745, 66

Muñoz-Mateos, J. C., Gil de Paz, A., Boissier, S., et al. 2007, ApJ, 658, 1006

Pan, Z., Li, J., Lin, W., Wang, J., \& Kong, X. 2014, ApJL, 792, L4

Panter, B., Jimenez, R., Heavens, A. F., \& Charlot, S. 2008, MNRAS, 391, 1117

Papaderos, P., Gomes, J. M., Vílchez, J. M., et al. 2013, A\&A, 555, L1

Peng, Y., Maiolino, R., \& Cochrane, R. 2015, Natur, 521, 192

Pérez, E., Cid Fernandes, R., González Delgado, R. M., et al. 2013, ApJL, 764, L1

Rees, M. J., \& Ostriker, J. P. 1977, MNRAS, 179, 541

Roche, N., Bernardi, M., \& Hyde, J. 2010, MNRAS, 407, 1231

Sánchez, S. F., Kennicutt, R. C., Gil de Paz, A., et al. 2012, A\&A, 538, A8

Sánchez, S. F., Pérez, E., Sánchez-Blázquez, P., et al. 2016a, RMxAA, 52, 21

Sánchez, S. F., Pérez, E., Sánchez-Blázquez, P., et al. 2016b, RMxAA, 52, 171

Sánchez, S. F., Rosales-Ortega, F. F., Iglesias-Páramo, J., et al. 2014, A\&A, 563, A49

Sánchez, S. F., Rosales-Ortega, F. F., Jungwiert, B., et al. 2013, A\&A, 554, A58

Sánchez-Blázquez, P., Rosales-Ortega, F. F., Méndez-Abreu, J., et al. 2014 A\&A, 570, A6

SDSS Collaboration, Albareti, F. D., Allende Prieto, C., et al. 2017, ApJS, 233, 25

Sheth, K., Melbourne, J., Elmegreen, D. M., et al. 2012, ApJ, 758, 136

Silk, J. 1977, ApJ, 211, 638

Simard, L., Mendel, J. T., Patton, D. R., Ellison, S. L., \& McConnachie, A. W. 2011, ApJS, 196, 11

Smee, S. A., Gunn, J. E., Uomoto, A., et al. 2013, AJ, 146, 32

Suh, H., Jeong, H., Oh, K., et al. 2010, ApJS, 187, 374

Szomoru, D., Franx, M., \& van Dokkum, P. G. 2012, ApJ, 749, 121

Szomoru, D., Franx, M., van Dokkum, P. G., et al. 2013, ApJ, 763, 73 
Toomre, A., \& Toomre, J. 1972, ApJ, 178, 623

Tortora, C., Napolitano, N. R., Cardone, V. F., et al. 2010, MNRAS, 407, 144

Tortora, C., Napolitano, N. R., Romanowsky, A. J., et al. 2011, MNRAS, 418, 1557

Tremonti, C. A., Heckman, T. M., Kauffmann, G., et al. 2004, ApJ, 613, 898

Vogt, N. P., Haynes, M. P., Giovanelli, R., \& Herter, T. 2004, AJ, 127, 3300

Wake, D. A., Bundy, K., Diamond-Stanic, A. M., et al. 2017, AJ, 154, 86

Wang, E., Kong, X., Wang, H., et al. 2017, ApJ, 844, 144

Weinmann, S. M., Kauffmann, G., van den Bosch, F. C., et al. 2009, MNRAS, 394, 1213

Wilkinson, D. M., Maraston, C., Thomas, D., et al. 2015, MNRAS, 449, 328
Willett, K. W., Lintott, C. J., Bamford, S. P., et al. 2013, MNRAS, 435, 2835 Wisnioski, E., Förster Schreiber, N. M., Wuyts, S., et al. 2015, ApJ, 799, 209 Wuyts, S., Förster Schreiber, N. M., Genzel, R., et al. 2012, ApJ, 753, 114

Wuyts, S., Förster Schreiber, N. M., Nelson, E. J., et al. 2013, ApJ, 779, 135 Yan, R., Bundy, K., Law, D. R., et al. 2016a, AJ, 152, 197

Yan, R., Tremonti, C., Bershady, M. A., et al. 2016b, AJ, 151, 8

Zhang, K., Yan, R., Bundy, K., et al. 2017, MNRAS, 466, 3217

Zhang, W., Li, C., Kauffmann, G., \& Xiao, T. 2013, MNRAS, 429, 2191

Zheng, Z., Wang, H., Ge, J., et al. 2017, MNRAS, 465, 4572

Zibetti, S., Charlot, S., \& Rix, H.-W. 2009, MNRAS, 400, 1181

Zibetti, S., Gallazzi, A. R., Ascasibar, Y., et al. 2017, MNRAS, 468, 1902 